\title{
The EREBOS project: Investigating the effect of substellar and low-mass stellar companions on late stellar evolution
}

\section{Survey, target selection, and atmospheric parameters}

\author{
V. Schaffenroth ${ }^{1}$, B. N. Barlow ${ }^{2}$, S. Geier ${ }^{1}$, M. Vučković3 ${ }^{3}$ D. Kilkenny ${ }^{4}$, M. Wolz ${ }^{5}$, T. Kupfer ${ }^{6}$, U. Heber $^{5}$, \\ H. Drechsel ${ }^{5}$, S. Kimeswenger ${ }^{7,8}$, T. Marsh ${ }^{9}$, M. Wolf $^{10}$, I. Pelisoli ${ }^{1}$, J. Freudenthal ${ }^{11}$, S. Dreizler ${ }^{11}$, \\ S. Kreuzer ${ }^{5}$, and E. Ziegerer ${ }^{5}$ \\ ${ }^{1}$ Institut für Physik und Astronomie, Universität Potsdam, Haus 28, Karl-Liebknecht-Str. 24/25, 14476 Potsdam-Golm, Germany \\ e-mail: schaffenroth@astro.physik.uni-potsdam.de \\ 2 Department of Physics, High Point University, One University Parkway, High Point, NC 27268, USA \\ 3 Instituto de Física y Astronomía, Facultad de Ciencias, Universidad de Valparaíso, Gran Bretaña 1111, Playa Ancha, \\ Valparaíso 2360102, Chile \\ 4 Department of Physics \& Astronomy, University of the Western Cape, Private Bag X17, Bellville 7535, South Africa \\ 5 Dr. Remeis-Observatory \& ECAP, Astronomical Institute, Friedrich-Alexander University Erlangen-Nürnberg, Sternwartstr. 7, \\ 96049 Bamberg, Germany \\ 6 Kavli Institute for Theoretical Physics, University of California, Santa Barbara, CA 93106, USA \\ 7 Institut für Astro- und Teilchenphysik, Universität Innsbruck, Technikerstrasse 25/8, 6020 Innsbruck, Austria \\ 8 Instituto de Astronomía, Universidad Católica del Norte, Av. Angamos 0610, Antofagasta, Chile \\ 9 Department of Physics, University of Warwick, Coventry CV4 7AL, UK \\ 10 Astronomical Institute, Faculty of Mathematics and Physics, Charles University, V Holešovičkách 2, 18000 Praha 8, \\ Czech Republic \\ 11 Institut für Astrophysik, Georg-August Universität Göttingen, Friedrich-Hund-Platz 1, 37077 Göttingen, Germany
}

Received 4 June 2019 / Accepted 19 July 2019

\begin{abstract}
Eclipsing post-common-envelope binaries are highly important for resolving the poorly understood, very short-lived commonenvelope phase of stellar evolution. Most hot subdwarfs $(\mathrm{sdO} / \mathrm{Bs})$ are the bare helium-burning cores of red giants that have lost almost all of their hydrogen envelope. This mass loss is often triggered by common-envelope interactions with close stellar or even substellar companions. Cool companions to hot subdwarf stars such as late-type stars and brown dwarfs are detectable from characteristic light-curve variations - reflection effects and often eclipses. In the recently published catalog of eclipsing binaries in the Galactic Bulge and in the Asteroid Terrestrial-impact Last Alert System (ATLAS) survey, we discovered 125 new eclipsing systems showing a reflection effect seen by visual inspection of the light curves and using a machine-learning algorithm, in addition to the 36 systems previously discovered by the Optical Gravitational Lesing Experiment (OGLE) team. The Eclipsing Reflection Effect Binaries from Optical Surveys (EREBOS) project aims at analyzing all newly discovered eclipsing binaries of the HW Vir type (hot subdwarf + close, cool companion) based on a spectroscopic and photometric follow up to derive the mass distribution of the companions, constrain the fraction of substellar companions, and determine the minimum mass needed to strip off the red-giant envelope. To constrain the nature of the primary we derived the absolute magnitude and the reduced proper motion of all our targets with the help of the parallaxes and proper motions measured by the Gaia mission and compared those to the Gaia white-dwarf candidate catalog. It was possible to derive the nature of a subset of our targets, for which observed spectra are available, by measuring the atmospheric parameter of the primary, confirming that less than $10 \%$ of our systems are not sdO/Bs with cool companions but are white dwarfs or central stars of planetary nebula. This large sample of eclipsing hot subdwarfs with cool companions allowed us to derive a significant period distribution for hot subdwarfs with cool companions for the first time showing that the period distribution is much broader than previously thought and is ideally suited to finding the lowest-mass companions to hot subdwarf stars. The comparison with related binary populations shows that the period distribution of HW Vir systems is very similar to WD+dM systems and central stars of planetary nebula with cool companions. In the future, several new photometric surveys will be carried out, which will further increase the sample of this project, providing the potential to test many aspects of common-envelope theory and binary evolution.
\end{abstract}

Key words. binaries: eclipsing - brown dwarfs - binaries: spectroscopic - binaries: close - subdwarfs - surveys

\section{Introduction}

Most subluminous B stars (sdBs) are core helium-burning stars with very thin hydrogen envelopes and masses around $0.5 M_{\odot}$ (Heber 2009, 2017). To form such an object, the hydrogen envelope of the red-giant progenitor must be stripped off almost entirely. Since a high fraction of sdB stars are members of short- period binaries (Maxted et al. 2001), common envelope ejection triggered by a close stellar companion is generally regarded as the most probable formation channel for many of the sdB stars.

There is however increasing evidence that substellar companions might also have a significant influence on sdB star formation (which is still poorly understood) and it has been proposed that planets and brown dwarfs could be responsible for 
the loss of envelope mass in the red-giant phase of sdB progenitors (Soker 1998). As soon as the primary star evolves to become a red giant, close substellar companions must enter a common envelope. Whether those objects are able to eject the envelope and survive, evaporate, or merge with the stellar core depends mostly on their mass. While planets below $10 M_{\text {Jup }}$ might not survive the interactions, companions exceeding this mass might be able to eject the envelope and survive as close companions (Soker 1998).

The best evidence for interactions with substellar companions is provided by the discovery of three close, eclipsing $\mathrm{sdB}$ binaries with brown dwarf companions. Photometric and spectroscopic follow-up observations of the sdB binary J162256+473051 revealed that the system is eclipsing with a period of $0.069 \mathrm{~d}$ and the companion is probably a brown dwarf with a mass of $0.064 M_{\odot}$ (Schaffenroth et al. 2014a). The short-period system $\mathrm{J} 082053+000843(0.096 \mathrm{~d})$ is also eclipsing, and the companion has a mass in the range $0.045-0.067 M_{\odot}$ (Geier et al. 2011). Schaffenroth et al. (2015) discovered another, especially interesting eclipsing hot subdwarf that shows pulsations and has a brown dwarf companion with a mass of $0.069 M_{\odot}$ (V2008$1753,0.065 \mathrm{~d})$. Additionally, two sdB systems with candidate brown dwarf companions have been detected (periods $\sim 0.3 \mathrm{~d}$, Schaffenroth et al. 2014b), but since they do not eclipse, only minimum companion masses - both below the hydrogen-burning limit - can be derived $\left(0.048\right.$ and $\left.0.027 M_{\odot}\right)$.

The most successful way to detect eclipsing binaries with cool stellar or substellar companions (HW Vir systems) is by inspecting their light curves, which, in addition to the eclipses, show a characteristic quasi-sinusoidal variation caused by the socalled reflection effect (see Schaffenroth et al. 2018, and references therein). This effect is observed in any close binary system consisting of a hot primary and a cool companion. As the secondaries in these systems are supposed to orbit synchronously, the hemisphere of the cool companion facing the hot primary is constantly irradiated, which leads to an increased flux over the orbital phase as the heated side of the secondary comes into view (see Wilson 1990; Budaj 2011, for a detailed discussion of this effect).

The amplitude of the reflection effect scales with the temperature ratio and the radii of the primary and secondary stars, as well as the inverse orbital separation (Wilson 1990; Budaj 2011). Hence, the reflection effect is strongest when both components of a close binary system have a very small separation, similar radii, and a high temperature difference. In systems consisting of a low-mass main sequence star or brown dwarf and a hot, compact star like a hot subdwarf, these conditions are fulfilled. The same features can be seen in very hot white dwarf binaries with cool companions, such as NN Serpentis (Parsons et al. 2010), or post-AGB stars with cool, main sequence companions, including central stars of planetary nebula (e.g., UU Sge, Afşar \& Ibanoğlu 2008).

Due to the short periods and similar radii of the components, sdB binaries with low-mass companions also have a high probability of eclipsing. Such systems are of great value because they allow the determination of the masses and radii of both components, as well as their separation, with an accuracy up to a few percent using combined photometric and spectroscopic analyses. The known sample of eclipsing and noneclipsing systems is relatively inhomogeneous, with most having been found in photometric surveys due to their characteristic light curve variations (e.g., Schaffenroth et al. 2013) or from light curves only covering a few hours aimed at searching for pulsations (e.g., Jeffery \& Ramsay 2014). These published systems have orbital periods between $0.069 \mathrm{~d}$ and $0.26 \mathrm{~d}$ (Schaffenroth et al. 2018, and references therein).

A photometric follow-up of spectroscopically selected targets (Schaffenroth et al. 2018) also allowed us to determine the fraction of substellar companions to sdBs. We derived a value of more than $8 \%$ for substellar companions in close orbits around sdBs. Moreover, they seem to be at least as frequent as low-mass stellar companions, as two of the four reflection-effect binaries we discovered are sdBs with substellar companions.

This shows that close substellar companions are able to eject a red-giant envelope and that they are much more frequent than predicted by standard binary-evolution theory. Due to their high fraction of close substellar companions, hot subdwarfs are well suited to the study of interactions between stars and brown dwarfs or giant planets. To understand both the common envelope phase under extreme conditions and the role of close-in planets and brown dwarfs for late stellar evolution, we need to study a large and homogeneously selected sample of eclipsing sdB binaries.

\section{Project overview}

The increasing number of photometric surveys provides us with a huge source to find more eclipsing hot subdwarf stars. Thirty-six new HW Vir candidates were discovered by the Optical Gravitational Lensing Experiment (OGLE) project (Pietrukowicz et al. 2013; Soszyński et al. 2015), almost tripling the number of such objects known and providing the first large sample of eclipsing sdBs. These systems have been identified by their blue colors, short orbital periods, and characteristic light curves in the $I$-band.

To investigate this unique sample of HW Vir candidates, we conduct the EREBOS (Eclipsing Reflection Effect Binaries from Optical Surveys) project, which aims to measure orbital, atmospheric, and fundamental parameters.

Key questions we want to answer over the course of the EREBOS project include the following: What is the minimum mass of the companion necessary to eject the common envelope? Is there a well-defined minimum mass or a continuum ranging from the most massive brown dwarfs down to hot Jupiter planets? What is the fraction of close substellar companions to sdBs and how does it compare to the possible progenitor systems such as main sequence stars with brown dwarf or hot Jupiter companions? To address these questions and understand both the common envelope phase under extreme conditions and the role of close-in planets and brown dwarfs for late stellar evolution, we need to know the parameters of post-commonenvelope systems over as much of the period distribution as possible.

In the following sections we discuss our target selection, try to constrain the nature of the primary star, and present the first results based on orbital parameters from the light curves and atmospheric parameters from the spectra we have taken so far.

\section{Target selection}

As already described, hot subdwarf binaries with cool, low-mass companions exhibit a unique light-curve shape produced by their strong eclipses and reflection effect. By visual inspection of light curves, the OGLE team identified 36 new HW Vir candidates in their sample (Pietrukowicz et al. 2013; Soszyński et al. 2015). In the course of the OGLE survey, approximately 450000 new eclipsing binaries have been published to date (Soszyński et al. 2016). More light-curve surveys monitoring billions of stars are 
available. As we are searching for systems with a defined set of characteristics, we developed a set of criteria to select a limited number of potential targets for our own visual inspection.

\subsection{Light-curve surveys}

We used two different surveys to search for light curves with the typical properties that we require.

\subsubsection{OGLE project}

OGLE is a long-term, large-scale photometric sky survey focused on variability. Its original purpose was the detection of micro-lensing events and it therefore focuses on observing fields with high stellar densities. A detailed description of the fourth phase of the project can be found in Udalski et al. (2015). OGLE-IV is conducted at the Las Campanas Observatory in Chile with a $1.3 \mathrm{~m}$ telescope dedicated to the project.

More than a billion sources are regularly monitored in different fields in the Galactic Bulge, the Small and Large Magellanic Clouds, and the Galactic disk. The OGLE-IV camera is equipped with $V$ - and $I$-band interference filter sets. The OGLE $I$-band filter very closely resembles the standard Johnson $I$-band filter; the OGLE-IV $V$-band filter is similar to the standard Johnson filter but extends slightly less far into the red. Most of the observations are performed in the $I$ filter and the resulting light curves have from several hundred to more than a thousand data points with an integration time of $100 \mathrm{~s}$. The OGLE-IV photometry covers the range of $12<I<20.5$ mag (Udalski et al. 2008). The light curves are published in different catalogs together with an ephemeris for each star.

\subsubsection{ATLAS project}

ATLAS is a high-cadence all-sky survey system designed to find dangerous near-Earth asteroids. ATLAS achieved first light in June 2015 and now consists of two independent units, one on Haleakala (HKO), and one on Mauna Loa (MLO) in the Hawai'ian islands. Details of the project can be found in Tonry et al. (2018). Each telescope is a $0.65 \mathrm{~m}$ Schmidt observing in a cyan filter ( $c$, covering $420-650 \mathrm{~nm}$ ) and an orange filter $(o, 560-820 \mathrm{~nm})$. The fisheye camera takes $32 \mathrm{~s}$ exposures on a $40 \mathrm{~s}$ cadence and ATLAS covers the entire accessible sky with a cadence of 2 days over a magnitude range $0<m<20$. As of the end of January 2018, ATLAS had taken about 600000 exposures resulting in 240 million light curves with more than 100 epochs.

\subsection{Color selection and period constraints}

Because sdBs are hot and therefore blue, we limited our search to the bluest targets in OGLE. As OGLE observes not only in the $I$-band but also in the $V$-band for most targets (more details in Sect. 3.1), we could use the color index $V-I$ for the selection. The Galactic Bulge is a very dense region and has very patchy and substantial reddening. Because of this, we cannot simply apply color cuts characteristic for hot subdwarfs. Instead, we investigated the colors of the HW Vir candidates identified by Pietrukowicz et al. (2013) and Soszyński et al. (2015) and decided to limit our search to targets with $V-I<1$ (see Fig. 1), as only three systems were found outside these limits. The longest-period HW Vir system previously known is AA Dor with a period of $0.26 \mathrm{~d}$. The longest-period reflectioneffect system has a period of $0.8 \mathrm{~d}$ (Jeffery \& Ramsay 2014).

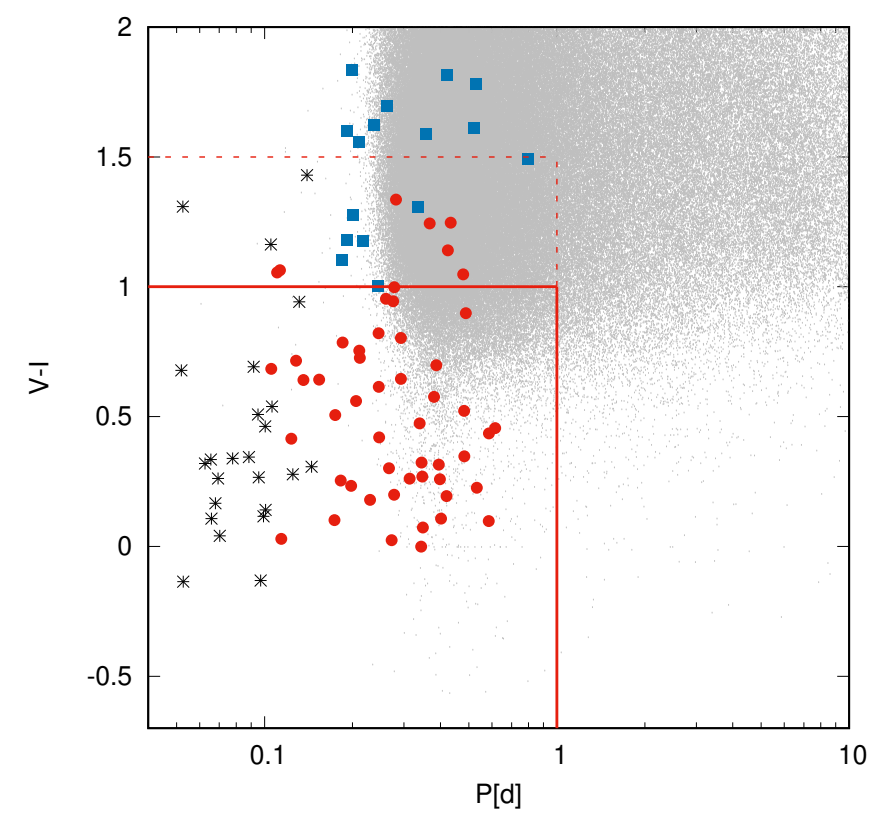

Fig. 1. Period and color selections applied to the eclipsing systems published by the OGLE team (Soszyński et al. 2016). HW Vir systems found by the OGLE team are shown as black stars. Red circles represent those found by visual inspection and blue squares those found by machine learning. It can be seen that we select short-period binaries with the bluest colors.

Consequently, we focused our search on systems with orbital periods less than one day. Later, we also started to extended our search up to $V-I<1.5$.

Figure 1 shows the color and period selection we applied and the systems we found. These criteria left us with 2200 systems, each of which was phased with the ephemeris provided by the OGLE team. We inspected the light curves of all 2200 systems visually and found 51 new HW Vir candidates with periods between 0.1 and $0.6 \mathrm{~d}$. Most of the other light curves were consistent with contact systems or $\beta$ Lyrae-type binaries. It is unsurprising that for the shortest periods almost all blue objects are HW Vir system candidates, as blue main sequence stars are much larger and have longer periods.

\subsection{Light-curve selection with machine learning}

To identify additional systems not covered by the initial color selection we used the light curves of the 87 identified systems as a training set for machine learning.

As this set already includes systems with a large period range, variety of inclinations, and different $\mathrm{S} / \mathrm{N}$, it is ideally suited for training. For this we used the support vector machine (SVM) provided by the PYTHON package SKLEARN. We performed a $C$-Support Vector Classification (SVC) with the default squared exponential (rbf) kernel with a penalty parameter, $C$, of $10^{5}$ and a Kernel coefficient, $\gamma$, of $10^{-2}$. All 450000 eclipsing binaries were phased with the OGLE ephemerides. The SVM was then trained with the previously identified systems and applied to all OGLE light curves.

Using the above procedure, 2613 light curves were selected. These were inspected again visually. In this way, 20 new systems were found, which have redder colors than our initial color selection. For two of these new systems, no observations are available in the $V$ band. Only six out of 87 systems found previously by our color criteria were not detected by the SVM. All of those 


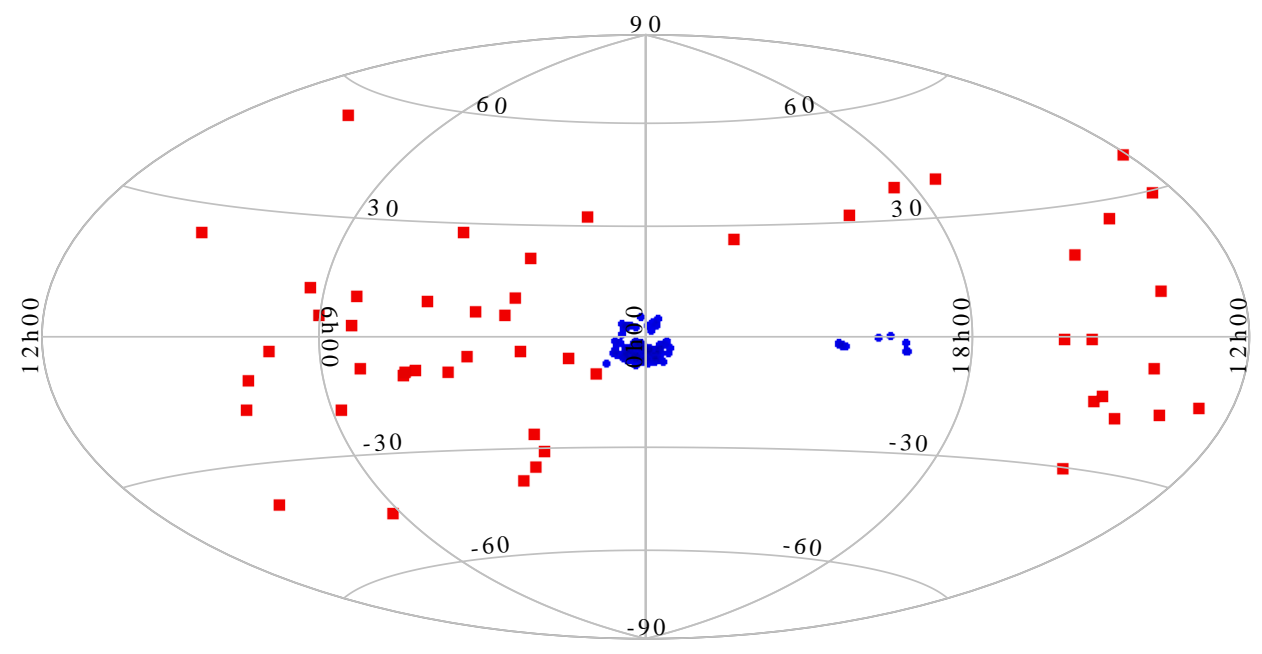

Fig. 2. Distribution of the OGLE targets (blue circles) and the ATLAS targets (red squares) on the sky. The OGLE fields in the Galactic disk and the Galactic Bulge are clearly visible. The ATLAS targets are distributed over the whole sky accessible from the northern hemisphere. systems have very poor $\mathrm{S} / \mathrm{N}$. This means the number of false positives is still relatively high, but we are dealing with a sufficiently small number of systems to investigate them all by eye. In the future we would like to improve this process by creating a sample of synthetic light curves with different inclinations, $\mathrm{S} / \mathrm{N}$, and orbital periods.

\subsection{Cross-matching the Gaia catalog of hot subdwarf stars with ATLAS}

Geier et al. (2019) published a catalog of candidate hot subdwarf stars of the complete sky. This catalog was used to search for more HW Vir systems by cross-matching it with ATLAS. With the cross-match, 1600 objects from the Gaia hot-subdwarf cata$\log$ were found in ATLAS. All of them were again phased after identifying the period with a Lomb-Scargle algorithm (Lomb 1976; Scargle 1982) and were then inspected visually. Fifty additional new HW Vir candidates were found in the ATLAS data, as well as several known systems. The light curves of all systems in our sample can be found in Figs. A.1 and A.2.

Figure 2 shows the Galactic distribution of the new HW Vir candidates. The OGLE and Gaia magnitudes of all our targets, along with those of the published HW Vir binaries, can be found in Tables A.1 and A.2. The OGLE targets, shown with blue circles, are found in the Bulge and the Galactic disk fields of OGLE. The ATLAS targets, shown with red squares, are distributed over the complete sky accessible from the Northern hemisphere.

The magnitude distribution of our targets is illustrated in Fig. 3. The OGLE targets peak at a brightness of 19 mag, which is much fainter than any previously known systems and makes the follow-up more difficult. However, we found several brighter systems in the ATLAS survey.

\section{Spectral parameters of the EREBOS targets}

We can obtain very accurate orbital parameters from the light curves: the orbital period, the inclination of the system, and the relative radii. However, the light curve analysis suffers from degeneracies due to several coupled parameters, and so it is essential to constrain as many of these parameters as possible using time-resolved spectroscopy. For 27 of our targets, we already have spectroscopic follow-up to confirm the nature of the primary star. All our observing runs can be found in Table 1. In the following we give more details of the spectroscopic

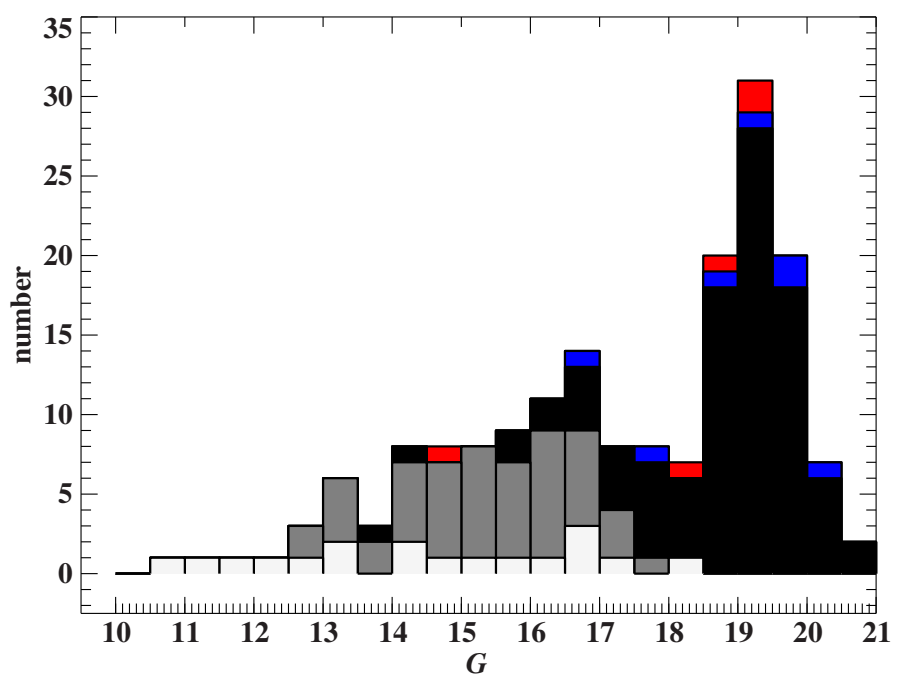

Fig. 3. Magnitude distribution of the published HW Vir systems (white), the ATLAS HW Vir candidates (gray), the OGLE HW Vir candidates (black), the eclipsing systems with candidate white dwarf primaries (blue) and the central stars of planetary nebula (red; more details are given in Sect. 5 and Tables A.1-A.3).

observations and show the first atmospheric parameters derived. Figure 4 shows three examples of our co-added spectra from the ESO-VLT/FORS2 spectrograph.

\subsection{Spectroscopic observations}

\subsubsection{ESO-NTT/EFOSC2}

For the brighter systems we used the EFOSC2 spectrograph mounted at the $3.58 \mathrm{~m} \mathrm{ESO/NTT}$ telescope. Nine of our systems have been observed in several runs (092.D-0040(A), 099.D0217(A), 0101.D-0791(A)). We always took several spectra per star in grism Gr\#19 (4441-5114 $\AA$ ) with a $1^{\prime \prime}$ slit to achieve a resolution of $R \sim 3000$ to derive a radial-velocity curve covering the whole orbit. This grism includes $\mathrm{H} \beta$ as well as HeI 4472 and 4922. Usually, several objects were observed in a repeating sequence but always with exposure times less than 5-10\% of the orbital period to avoid orbital smearing. To derive the atmospheric parameters, moreover, one spectrum per star was taken with grism Gr\#07 (3270-5240 ̊) with resolution $R \sim 500-700$. This grism covers the Balmer jump. 
Table 1. Spectroscopic follow-up observations.

\begin{tabular}{llllc}
\hline \hline Date & Nights & Run & Telescope and instrument & Observers \\
\hline 09/10 May 2015 & 2 & 095.D-0167(A) & ESO-VLT/FORS2 & S. Kimeswenger \\
Oct. 2015-Jun. 2017 & 12.5 & 196.D-0214(A-D) & ESO-VLT/FORS2 & Service \\
04/05 Aug. 2016 & 2 & NOAO 2016B-0283 & SOAR/Goodman & B. Barlow \\
30/31 Mar. 2016 & 2 & NOAO 2016A-0259 & SOAR/Goodman & B. Barlow \\
07/08 Jun. 2016 & 2 & NOAO 2016A-0259 & SOAR/Goodman & B. Barlow \\
01-05 Feb. 2014 & 4 & 092.D-0040(A) & ESO-NTT/EFOSC2 & S. Geier \\
01-04 Jul. 2017 & 3 & 099.D-0217(A) & ESO-NTT/EFOSC2 & E. Ziegerer \\
30 Jul.-02 Aug. 2018 & 3 & 0101.D-0791(A) & ESO-NTT/EFOSC2 & S. Kreuzer \\
26 Feb. 2019 & 1 & SO2018B-002 & SOAR/Goodman & I. Pelisoli \\
\hline
\end{tabular}

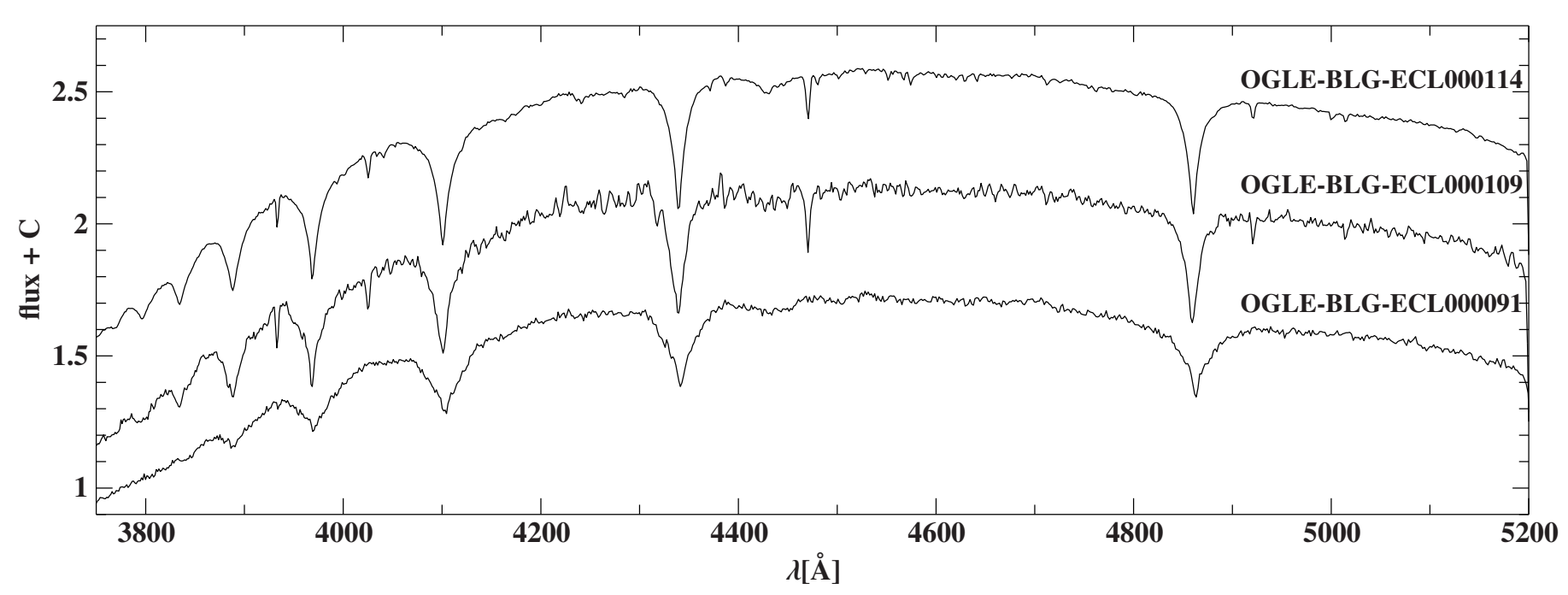

Fig. 4. Three example spectra showing a typical sdB (OGLE-BLG-ECL-000114), a DA white dwarf (OGLE-BLG-ECL-000091), and a pre-He white dwarf (OGLE-BLG-ECL-000109). SdBs and pre-He white dwarfs show very similar spectra and can only be distinguished by deriving the atmospheric parameters.

\subsubsection{ESO-VLT/FORS2}

Most of the OGLE target periods are quite short and, as we are interested especially in the shortest periods, this limits the exposure times greatly; EFOSC2 is not adequate for observation of those targets. We applied successfully for an ESO large program with ESO-VLT/FORS (196.D-0214(A-D)) for fainter, short-period systems, after a feasibility study in visitor mode (095.D-0167(A)). In our first run on 10 May 2015 we observed one 20-mag object (OGLE-GD-ECL-10384) for a half a night taking 24 spectra using Grism GRIS_600B+22 (330-621 A, 1" slit, $R \sim 780$ ) each with an exposure time of $600 \mathrm{~s}$ and covering two full orbits. The ESO large program was executed in service mode over the course of two years. We divided all observations into 1 hour observing blocks (OBs), in which time-resolved spectroscopy of one target was performed per block. As the periods of many of our targets are very short, a significant part of the orbit can be covered in one hour. For these observations we used Grism GRIS_1200B+97 (3660-5110, 1" slit, $R \sim 1420)$. We limited the exposure time to $\sim 5 \%$ of the orbital period to prevent orbital smearing. About 6-8 spectra were taken per OB. For each target, several OBs were taken to cover the whole orbit distributed over one semester.

The observation of the Bulge targets and the data analysis turned out to be less straightforward than expected, as we have problems with the enormous crowding in the Bulge field, which complicates photometry as well as spectroscopy. Already, the identification of the targets was a huge challenge at the beginning, because of the lack of good finding charts in the visual, which lead to misidentification of the target in some cases. Additionally, the ESO-VLT/FORS2 spectrograph was found not to be as stable as expected, because it was not designed for the determination of radial velocities. This complicates the determination of accurate radial velocity curves. Hence, we concentrate only on the determination of the atmospheric parameters for this paper, as more work is needed to derive the radial velocity curves from the FORS2 spectra. The radial velocities of all of our targets will be presented in future papers.

Individual spectra were then co-added after being corrected for radial velocity so that we could use the co-added spectra for atmospheric analyses. Radial velocities were determined with the IRAF ${ }^{1}$ task FXCORR for cross-correlation against model spectra and co-added spectra to measure the radial velocity shifts in the Balmer and helium lines.

\subsubsection{SOAR/Goodman}

We have collected time-resolved spectroscopy of many of the brighter, short-period systems with the Goodman Spectrograph on the $4.1 \mathrm{~m}$ SOuthern Astrophysical Research (SOAR) telescope (Clemens et al. 2004). Four of our targets have been observed to date with time allocated through the National

\footnotetext{
1 Tody (1986).
} 
Table 2. Atmospheric parameters of our observed targets from the EREBOS project spectroscopy with statistical errors.

\begin{tabular}{lccc}
\hline \hline Target name & $\begin{array}{c}T_{\text {eff }} \\
{\left[10^{3} \mathrm{~K}\right]}\end{array}$ & $\begin{array}{c}\log g \\
{[\mathrm{cgs}]}\end{array}$ & $\log y$ \\
\hline OGLE-BLG-ECL-000114 $^{(a)}(c)$ & $29.2 \pm 0.5$ & $5.55 \pm 0.07$ & $-2.28 \pm 0.10$ \\
OGLE-BLG-ECL-000139 $^{(c)}$ & $29.6 \pm 0.5$ & $5.50 \pm 0.06$ & $-2.97 \pm 0.13$ \\
OGLE-BLG-ECL-000103 $^{(a)}$ & $29.5 \pm 0.4$ & $5.70 \pm 0.05$ & $-1.75 \pm 0.11$ \\
OGLE-BLG-ECL-000163 $^{(c)}$ & $28.0 \pm 0.3$ & $5.37 \pm 0.04$ & $-1.96 \pm 0.10$ \\
OGLE-BLG-ECL-000124 $^{(a)}$ & $26.3 \pm 0.7$ & $5.46 \pm 0.06$ & $-2.02 \pm 0.16$ \\
OGLE-BLG-ECL-000010 $^{(a)}$ & $27.9 \pm 0.7$ & $5.32 \pm 0.06$ & $-2.59 \pm 0.18$ \\
OGLE-BLG-ECL-000202 $^{(a)}$ & $36.3 \pm 0.5$ & $5.25 \pm 0.09$ & $-3.17 \pm 0.12$ \\
OGLE-BLG-ECL-000110 $^{(a)}$ & $23.6 \pm 0.5$ & $5.35 \pm 0.05$ & $-2.30 \pm 0.13$ \\
OGLE-BLG-ECL-000109 $^{(a)}$ & $29.3 \pm 0.3$ & $6.05 \pm 0.05$ & $-1.96 \pm 0.10$ \\
OGLE-BLG-ECL-000212 $^{(a)}$ & $30.1 \pm 0.5$ & $5.38 \pm 0.07$ & $-2.64 \pm 0.11$ \\
OGLE-BLG-ECL-000207 $^{(a)}$ & $24.4 \pm 0.4$ & $5.54 \pm 0.06$ & $-2.15 \pm 0.14$ \\
OGLE-BLG-ECL-173411 $^{(b)}$ & $26.0 \pm 0.1$ & $5.28 \pm 0.03$ & $-2.48 \pm 0.11$ \\
OGLE-BLG-ECL-361688 $^{(b)}$ & $27.2 \pm 1.3$ & $5.20 \pm 0.08$ & $-2.39 \pm 0.02$ \\
OGLE-BLG-ECL-416194 $^{(b)}$ & $35.6 \pm 0.9$ & $5.34 \pm 0.20$ & $-2.21 \pm 0.35$ \\
OGLE-BLG-ECL-017842 $^{(b)}$ & $29.7 \pm 0.8$ & $5.81 \pm 0.17$ & $-2.65 \pm 0.28$ \\
OGLE-BLG-ECL-280838 $^{(b)}$ & $28.3 \pm 0.6$ & $5.55 \pm 0.08$ & $-2.78 \pm 0.25$ \\
OGLE-BLG-ECL-412658 $^{(b)}$ & $36.7 \pm 0.8$ & $5.48 \pm 0.08$ & $-2.49 \pm 0.33$ \\
OGLE-GD-ECL-08577 $^{(b)}$ & $28.4 \pm 1.0$ & $5.43 \pm 0.15$ & $-2.01 \pm 0.27$ \\
OGLE-GD-ECL-10834 $^{(a)}$ & $27.6 \pm 0.8$ & $5.64 \pm 0.16$ & $-2.54 \pm 0.18$ \\
OGLE-GD-ECL-11388 $^{(b)}$ & $29.0 \pm 0.3$ & $5.56 \pm 0.04$ & $-2.77 \pm 0.05$ \\
OGLE-GD-ECL-11471 $^{(a)}$ & $28.4 \pm 0.5$ & $5.71 \pm 0.10$ & $-2.17 \pm 0.08$ \\
\hline J282.4644-13.6762 $^{(b)}$ & $27.5 \pm 0.6$ & $5.54 \pm 0.07$ & $-2.25 \pm 0.21$ \\
J351.7186+12.5060 $^{(b)}$ & $29.0 \pm 0.4$ & $5.68 \pm 0.08$ & $-1.86 \pm 0.14$ \\
J315.0724-14.190 $^{(b)}$ & $30.2 \pm 2.0$ & $5.96 \pm 0.38$ & $-2.00 \pm 0.16$ \\
J079.5290-23.1458 $^{(b)}$ & $30.9 \pm 0.7$ & $5.75 \pm 0.09$ & $-1.96 \pm 0.14$ \\
J129.0542-08.0399 $^{(c)}$ & $31.1 \pm 0.3$ & $5.49 \pm 0.06$ & -2.85 \\
\hline & & & \\
\hline
\end{tabular}

Notes. ${ }^{(a)}$ ESO-VLT/FORS2. ${ }^{(b)}$ ESO-NTT/EFOSC2. ${ }^{(c)}$ SOAR/Goodman.

Optical Astronomy Observatory (NOAO Proposal IDs 2016B0283, 2016A-0259,SO2018B-002). Our standard observing configuration uses a $930 \mathrm{~mm}^{-1}$ VPH grating from Syzygy Optics, LLC, in conjunction with a $1.03^{\prime \prime}$ longslit to achieve a spectral resolution of $2.9 \AA(R \sim 1500)$ over the wavelength range 3600 $5300 \AA$. We observed each target using a series of consecutive spectra covering one full orbital period. In order to maximize our duty cycle, the spectral images were binned $2 \times 2$, resulting in a pixel scale of $0.3^{\prime \prime}$ per binned pixel in the spatial direction and $0.84 \AA$ per binned pixel in the dispersion direction. We again kept the integration times to less than $5 \%$ of the orbital period to avoid phase smearing. We use Gaussian fits to the Balmer lines to determine radial velocities and created a co-added spectrum for atmospheric modeling after individually correcting their velocities.

\subsection{Atmospheric parameters}

Atmospheric parameters were determined by calculating synthetic spectra using LTE model atmospheres with solar metallicity and metal line blanketing (Heber et al. 2000) and fitting these to the Balmer and helium lines using SPAS (Hirsch 2009). In this way, we determined the atmospheric parameters (effective temperature $T_{\text {eff }}$, surface gravity $\log g$ and helium abundance $\log y$ ) for 25 systems, which can be found in Table 2. Some example line fits are showed in Fig. 5. The errors given are only statistical errors. As the contribution of the companion to the visible flux varies over the orbital phase due to the reflection effect, apparent variations of the atmospheric parameters are found in some eclipsing sdB binaries with cool companions with high-S/N spectra on the order of $1500 \mathrm{~K}$ and 0.1 dex over the whole orbital phase (Schaffenroth et al. 2014a). This means that in the case of systems with very low statistical error we have to adopt an uncertainty of $750 \mathrm{~K}$ in temperature and $0.05 \mathrm{dex}$ in $\log (g)$.

The parameters show that the primaries are mostly typical for HW Vir systems (see Schaffenroth et al. 2018, and references therein). OGLE-BLG-ECL-000091 and J186.9106-30.7203 are the only exceptions, as both clearly look like DA white dwarfs with very broad Balmer lines (see Fig. 4 for an example).

However, it is not only the tracks of He-core burning objects that cross the extreme horizontal branch, but also those of postRGB objects with masses too low to burn $\mathrm{He}$ in the core (see Fig. 6). Both of the above evolve directly into He-WD and are therefore also called pre-He WDs. The lifetime of pre-He WDs crossing the EHB is only one hundredth of the lifetime of a coreHe-burning sdB on the EHB. Therefore, we expect them to be much rarer and that most objects on the EHB are hot subdwarfs. This also depends, however, on the birth rate of these systems, which is unknown. This means in most cases that it is not possible to distinguish between sdBs and pre-He WDs from the atmospheric parameters alone.

To compare the atmospheric parameters of our targets to other $\mathrm{sdB}$ binaries we plotted the $T_{\text {eff }}-\log g$ diagram of the reflection-effect systems and other sdB binaries from Kupfer et al. (2015) in Fig. 6. The published HW Vir and reflection-effect systems seem to cluster in a relatively small region of the $T_{\text {eff }}-\log g$ diagram. Very few are found on the EHB at higher temperatures. Our new systems almost double the number of atmospheric parameter determinations for sdBs with cool, low-mass companions. It appears now that the EHB is well populated with such systems. It is only near the He-main sequence (HeMS) that we still do not find any HW Vir systems. Moreover, we find three systems which have already evolved away from the EHB.

An interesting system is OGLE-BLG-ECL-000109, which lies clearly below the He-main sequence. This means it cannot be a He-core burning object is most probably a post-RGB object with a mass that is too low to burn He in the core. The position of OGLE-BLG-ECL-000109 agrees best with a track for a very low-mass white dwarf with a mass near $0.27 M_{\odot}$ (Althaus et al. 2013). Some HW Vir systems have been found where the analysis could not unambiguously distinguish between a helium-core-burning object on the extreme horizontal branch and a pre-He WD (e.g., J082053+000843 and HS 2231+2441, Geier et al. 2011; Almeida et al. 2017). This is the first HW Vir system lying significantly below the EHB and can only be explained by being a pre-He white dwarf.

\section{Constraining the nature of the primary star}

We selected our candidates purely based on the shapes of their light curves. To learn about the population of our targets, we need to constrain the nature of the primary star. The easiest way to do this is with spectroscopy and so far we have spectroscopic confirmation for 25 systems with a most likely hot subdwarf primary. Only two of our targets have a white dwarf primary (see Sect. 4.2).

For the rest of our sample, we have not yet obtained spectroscopy. As described in Sect. 2, white dwarfs or post-AGB objects can be possible contaminants in our sample. Of course, such systems are no less interesting, but it is important to have a homogeneous sample from which to draw conclusions.

The ESA Gaia Data Release 2 (Gaia Collaboration 2018b) provided us for the first time with tools to constrain the nature of the primary star. All results and Gaia parameters for our systems 

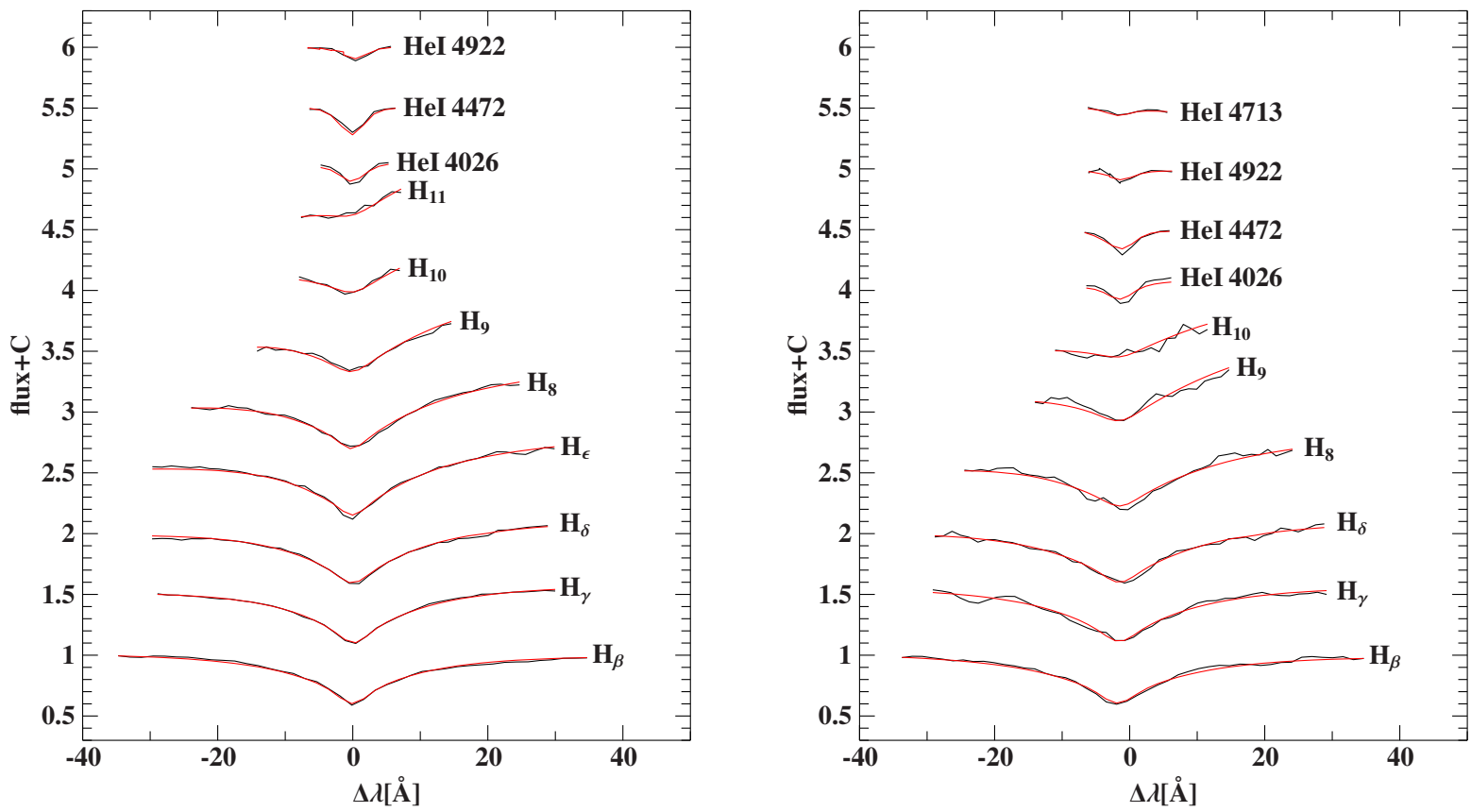

Fig. 5. Example fits of hydrogen and helium lines with model spectra for a typical sdB (OGLE-BLG-ECL-000103, left panel), and a pre-He WD star (OGLE-BLF-ECL-000109, right panel). The atmospheric parameters of these stars are given in Table 2.

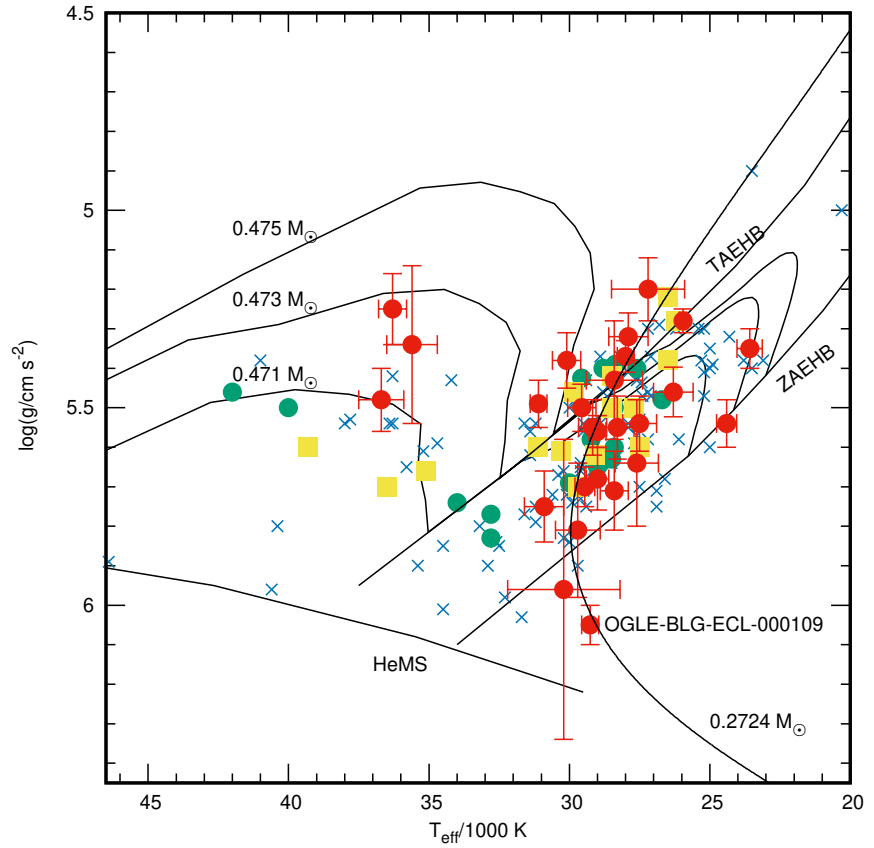

Fig. 6. $T_{\text {eff }}-\log g$ diagram of the HW Virginis systems. The zero-age EHB and ZAEHB, and the terminal-age EHB and TAEHB are superimposed by evolutionary tracks by Dorman et al. (1993) for sdB masses of $0.471,0.473$, and $0.475 M_{\odot}$ with one track for an extremely low-mass white dwarf of a mass of $0.2724 M_{\odot}$ by Althaus et al. (2013). The newly found systems are shown as red circles with error bars. The published HW Vir systems are shown as green circles, the reflection effect systems without eclipses as yellow squares. The blue crosses represent other sdB binaries (Kupfer et al. 2015), either with white dwarf or unknown companions.

can be found in Tables A.1, A.2, A.4, and A.5. Parallaxes and proper motions are available for 75 of the 107 OGLE targets and all ATLAS targets have.

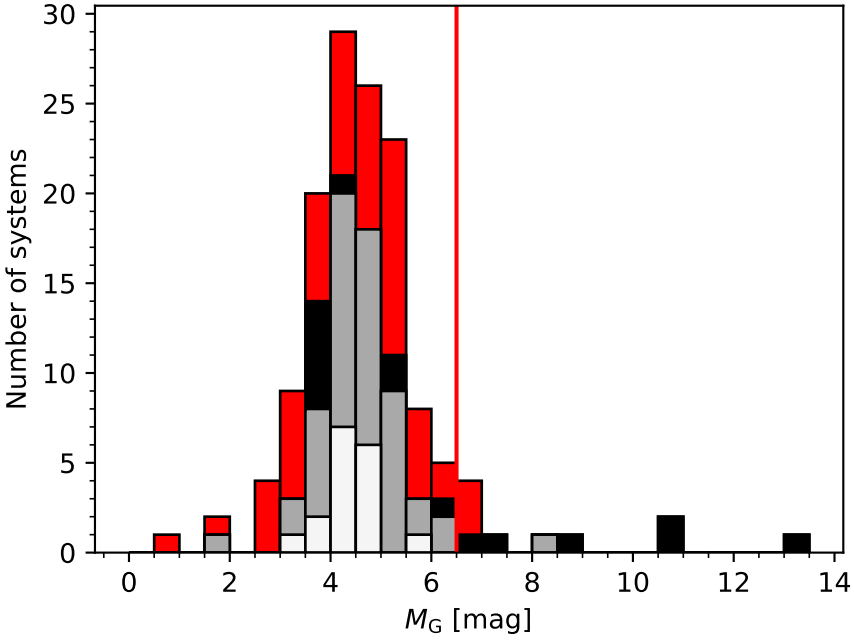

Fig. 7. Distribution of the absolute $G$ magnitude $\left(M_{G}\right)$ of our targets. The published HW Vir stars are shown in white; the ATLAS targets are displayed in gray; and the OGLE targets with parallaxes with errors less than $25 \%$ are displayed in black. For the rest of the OGLE targets we used the distances by Bailer-Jones et al. (2018), shown in red. The red line marks $M_{G}=6.5 \mathrm{mag}$, above which the primary stars are faint enough that they are more likely to be white dwarfs.

\subsection{Absolute magnitudes and distances}

As seen in Fig. 6 it is not possible to uniquely distinguish sdBs from pre-He WDs from the atmospheric parameters alone. However, the masses and radii of pre-He WDs are expected to be lower, and therefore, the absolute magnitudes are fainter. Hot subdwarf stars have radii around $0.15-0.2 R_{\odot}$, comparable to $\mathrm{M}$ dwarf stars. White dwarfs have radii that are much smaller comparable to that of Earth $\left(\sim 0.01 R_{\odot}\right)$ - and usually have much fainter absolute magnitudes.

In Fig. 7 the distribution of the absolute $G$ magnitude $\left(M_{G}\right)$ is shown. Absolute magnitudes were calculated via the distance 


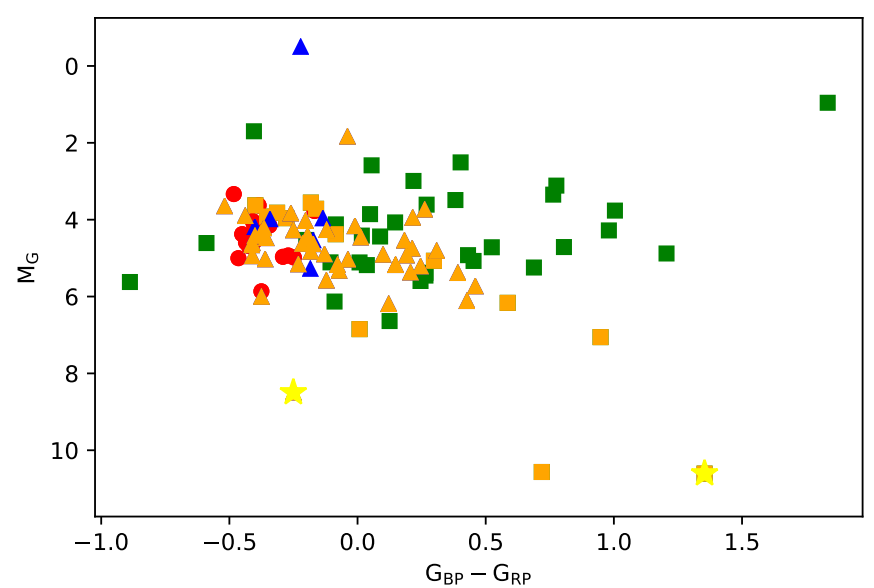

Fig. 8. Color-magnitude diagram of all our targets in Gaia colors. Red circles represent the published HW Vir systems for comparison. Squares indicate the OGLE targets and triangles the ATLAS targets. Targets with parallaxes better than $25 \%$ are marked in orange. For the green OGLE targets and the blue ATLAS targets we used the distances by Bailer-Jones et al. (2018). The yellow stars mark our confirmed system with a white dwarf primary.

modulus $G-M_{G}=5 \log _{10} d-5+\left(A_{G}\right)$. We used the distances published by Bailer-Jones et al. (2018). Distances with large parallax errors are mostly based on the length scale model they use, which is unclear for sdBs, and therefore they have to be taken with caution. As all our systems show photometric variability, the uncertainty in $G$ will also be larger than that given in the DR2 Gaia catalog. On the other hand, outliers are neglected for the determination of $G$, and therefore the mean Gaia magnitude should give a good estimate of the correct sdB magnitude (Riello et al. 2018).

For targets in the Bulge, the reddening cannot be neglected, and we constrained the reddening $E(B-V)$ by using Stilism $^{2}$ (Lallement et al. 2014), which gives the reddening at certain coordinates depending on the distance. In most cases, we derive only a lower limit for the reddening because the dust maps do not extend far enough $(<1-2 \mathrm{kpc})$. To obtain the Gaia $G$-band extinction coefficient $A_{G}$ we used Eq. (1) from the Gaia Collaboration (2018a), which uses $G, \mathrm{BP}, \mathrm{RP}$, and $E(B-V)$ as input parameters. For some of the OGLE targets, no BP or RP was given in the Gaia data, so we assumed similar colors to those found for the other OGLE targets (see Fig. 8).

When looking at the distribution of absolute $G$ magnitudes of all targets, it is obvious that the different subsamples show the same distribution peaking at $M_{G}=4.5 \mathrm{mag}-$ as expected for sdBs. We checked the absolute magnitudes of the white dwarf candidates from the Gaia white dwarf catalog (Gentile Fusillo et al. 2019) and the Gaia hot subdwarf catalog (Geier et al. 2019). Most of the objects with $M_{G}>6.5$ are classified as white dwarf stars, and therefore a white dwarf primary is more likely. Nine of our objects have absolute magnitudes fainter than $6.5 \mathrm{mag}$ and two of them have been spectroscopically confirmed as white dwarfs. On the other hand, three of them have been confirmed as sdBs. As mentioned before, the reddening we applied is only a lower limit, and therefore the absolute magnitude of the OGLE targets is an upper limit. The temperatures of those three targets is relatively low for sdBs with $24000-26000 \mathrm{~K}$. It is also possible that they are pre-He WDs instead of sdBs.

\footnotetext{
2 https://stilism.obspm.fr/
}

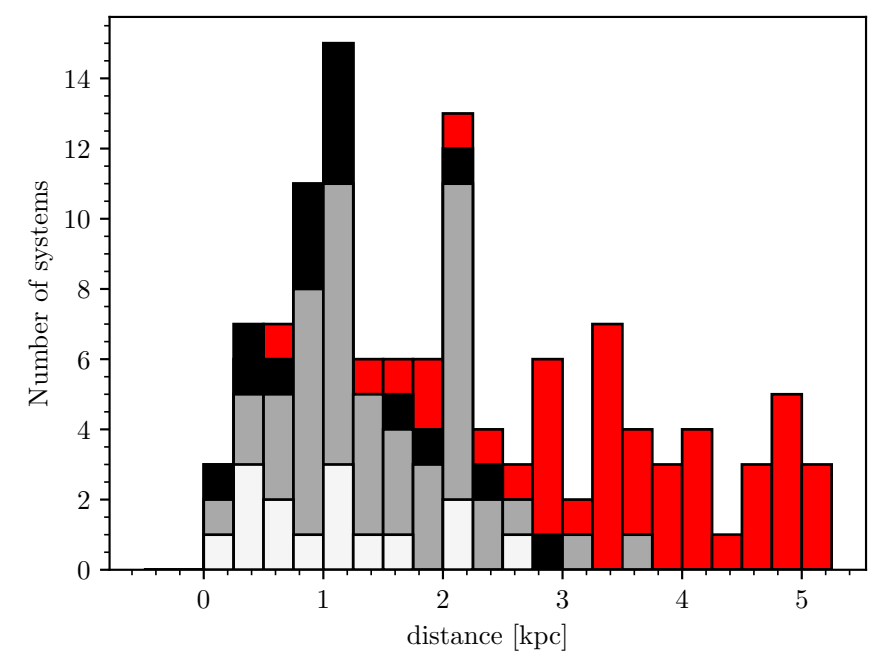

Fig. 9. Distance distribution of our targets. The published HW Vir stars are shown in white, the ATLAS targets are displayed in gray, and the OGLE targets with parallaxes that have errors of less than $25 \%$ are displayed in black. For the rest of the OGLE targets, shown in red, we used the distances by Bailer-Jones et al. (2018).

Another criterion is the color of the targets, which we can combine with absolute magnitude in a color magnitude diagram, shown in Fig. 8. The published HW Vir stars (see Tables A.1 and A.4) are concentrated at BP $-\mathrm{RP}<0$. The ATLAS targets show a larger spread, but we neglected reddening for those, which might be important for more distant targets. Due to the high reddening, which can only be poorly constrained in the bulge, the OGLE targets have an even wider distribution in color.

From the Gaia parallaxes it is also possible to determine the distances of the objects; this is shown in Fig. 9. The distance distribution agrees nicely with the distribution of the Gaia hot subdwarf catalog (Geier et al. 2019). The only difference is that there appear to be anomalously few objects observed at distances between $1.25 \mathrm{kpc}$ and $2 \mathrm{kpc}$. This bi-modality is mainly seen in the ATLAS targets. As expected from their fainter magnitudes, the OGLE targets are mostly further away (80\% have distances $>2.5 \mathrm{kpc}$ ). All but two targets with distances larger than $3 \mathrm{kpc}$ have large parallax errors and hence uncertain distances.

\subsection{Reduced proper motions}

As the reddening cannot be constrained perfectly for the moredistant objects, and because they have parallaxes with large errors, the absolute magnitudes of these targets are not reliable. However, to distinguish hot white dwarfs from hot subdwarf stars, reduced proper motions can be used. The reduced proper motion, defined as $H_{G}=G+5(\log \mu+1)$, can be used as a proxy for the distance of an object, since more-distant objects exhibit less movement, on average. Gentile Fusillo et al. (2015) showed that this selection method is well suited to separate hot subdwarfs from white dwarf candidates.

Figure 10 shows the distribution of the reduced proper motions of our targets superimposed with the distribution for white dwarfs taken from the Gaia white dwarf catalog (Gentile Fusillo et al. 2019). It is clear that the subdwarfs are found at smaller reduced proper motions. However, there is a region where both distributions overlap and the nature of the primary cannot be determined unambiguously. Combining the selection from the absolute magnitude with the reduced proper 


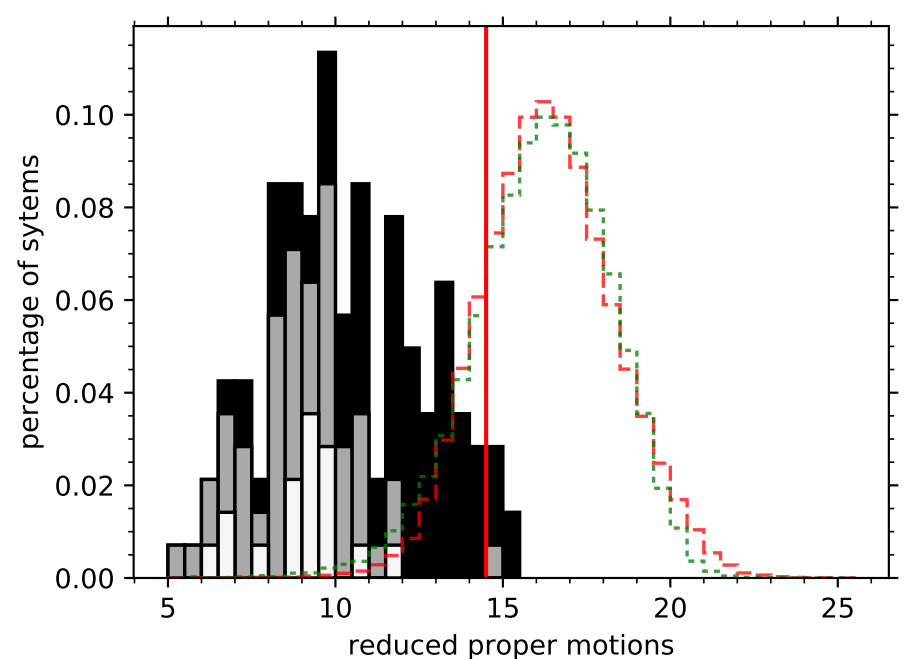

Fig. 10. Normalized distribution of the reduced proper motions of our targets (white are the published HW Vir systems, gray the ATLAS targets, and black the OGLE targets). Superimposed are the reduced proper motions of the objects with a white dwarf probability greater than $90 \%$ from the Gaia white dwarf catalog (Gentile Fusillo et al. 2019), shown with the red dashed line. The green dotted line shows the subsample of the targets with spectral classification from SDSS spectra. The red line marks our reduced proper motion cut of $H_{G}<14.5$; at higher values, targets are less likely to be hot subdwarfs.

motion and the spectroscopically confirmed targets, we decided to define the cut at $H_{G}<14.5$. This leaves us with seven objects, which have higher probabilities of being white dwarf binaries with cool, low-mass companions out of 123 objects with Gaia parallaxes and proper motions. All white dwarf binary candidates can be found in Tables A.2 and A.5. This means that the white dwarf binaries represent only a very small part of our sample and most targets are indeed hot subdwarf binaries.

This becomes even more clear when we look at the relation between reduced proper motion and apparent $G$ magnitude (Fig. 11). The bulk of white dwarfs is seen at larger reduced proper motions than the sdBs and also at fainter apparent magnitudes. It is not surprising that most white dwarfs are fainter as they have much smaller radii. Only very few white dwarfs are found in the overlapping region. However, they are much more frequent, and so an uncertainty for some OGLE targets remains.

\subsection{Binary central stars of planetary nebula}

Another class of objects that can have similar light curves to those of HW Vir systems are binary central stars of planetary nebula (bCSPNs) with cool, low-mass companions. For those objects, whether the nebulae are ejected AGB or RGB envelopes, or ejected common envelopes (e.g., Hillwig et al. 2017) is under debate. With our selection criteria we cannot properly distinguish bCSPNs from sdBs. However, bCSPNs have relatively short lifetimes and are therefore much rarer (Miller Bertolami 2016). In our absolute magnitude distribution, we have a few targets which are much brighter than the rest. For the latter, the probability of being a post-AGB binary with an extremely hot primary is higher. OGLE-BLG-ECL-412658 with an absolute magnitude of $1.69_{-0.98}^{+0.92}$ was found to be an sdB star with a temperature of $35600 \mathrm{~K}$ evolving away from the EHB. Two of our targets (J265.3145+29.5881 and OGLE-BLGECL-149869) seem to have an absolute magnitude brighter than $1 \mathrm{mag}$, which is much brighter than one would expect for a hot

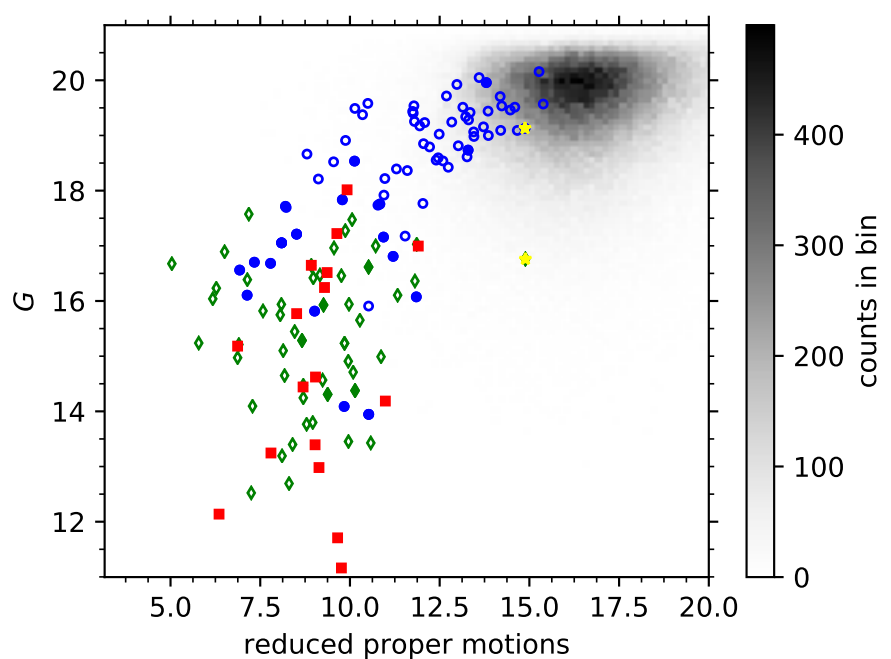

Fig. 11. Relation between reduced proper motion and apparent $G$ magnitude. Filled symbols mark objects with spectral confirmation. Red squares represent the published HW Vir systems, green diamonds the ATLAS targets, and blue circles the OGLE targets. The yellow star marks the confirmed white dwarf binary. Superimposed is the number distribution of objects with a white dwarf probability greater than $90 \%$ from the Gaia white dwarf catalog (Gentile Fusillo et al. 2019).

subdwarf. However, both have a very small and uncertain parallax. J171.4930-20.1447 on the other hand has an absolute magnitude of $1.66 \pm 0.22$ with a small parallax error of only $10 \%$ and is therefore the best candidate to be a post-AGB object.

Miszalski et al. (2009) performed a survey to find planetary nebulae in the direction of the Galactic Bulge. Four objects from our target list were confirmed as planetary nebulae by these latter authors; these can be found in Tables A.3 and A.6. Another known planetary nebula was found in the cross-match of the Gaia hot subdwarf catalog with ATLAS. Currently only 11 eclipsing central stars of planetary nebula showing a reflection effect are known ${ }^{3}$.

\subsection{Nature of the primary star}

By combining all criteria, it is safe to say that we have only a small level of contamination by white dwarfs or post-AGB binaries, probably less than $10 \%$. For most of our target sample, an $\mathrm{sdB}$ primary star is most likely. This is also supported by the fact that only 2 out of 28 targets were confirmed not to have an $\mathrm{sdB}$ primary (see Sect. 4.2).

\section{Results}

\subsection{The period distribution of eclipsing hot subdwarfs with cool companions}

With all the newly discovered candidate HW Vir systems presented here, the number of known systems has increased from 20 to 170 . The most straightforward parameter to derive from light curves of eclipsing binaries is the orbital period. It can be found in Table A.1. All light curves are displayed in Figs. A.1 and A.2. The period distribution is shown in Fig. 12. The previously known period distribution of the HW Vir systems covered a range of $0.07-0.26 \mathrm{~d}$ with a sharp peak around $0.1 \mathrm{~d}$; the

http://www.drdjones.net/bCSPN/ 


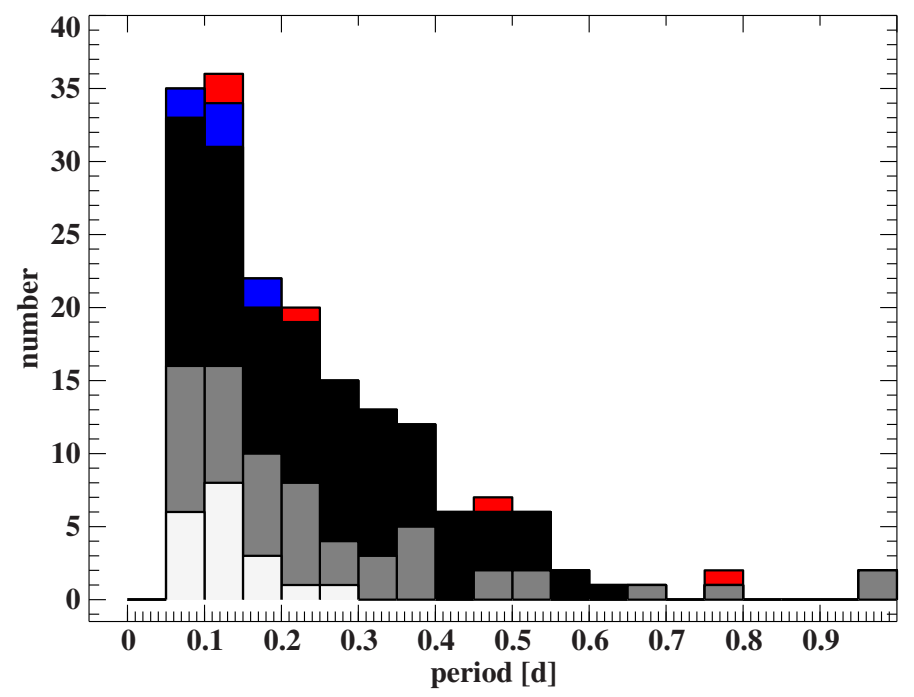

Fig. 12. Period distribution for all our targets. The currently published HW Vir systems are shown in white, with the ATLAS targets in gray, and the OGLE targets in black. In blue we marked those systems which have a higher probability of being white dwarf binaries, in red the central stars of planetary nebula.

distribution of the new systems is much broader, now spanning periods from just $0.05 \mathrm{~d}$ to more than one day.

As the eclipsing probability strongly correlates with the period, it is not surprising that most systems are found at periods around $0.1 \mathrm{~d}-$ as seen in the smaller sample. The number of systems at shorter periods has increased, but we also find a significant number at longer periods - up to $0.5 \mathrm{~d}$. This population was previously completely unknown, but most of the known systems were found while looking for short-period pulsations with light curves of perhaps only an hour or two, and therefore longperiod systems would not necessarily have been detected. Apart from the smaller chance of detecting eclipses, reflection effects will tend to become much smaller as the component separation and period increase.

As we limit ourselves to eclipsing systems, we also tried to constrain the true period distribution. For this we had to correct for the number of systems which are not selected by EREBOS because they do not show eclipses. The probability of eclipses occurring is dependent on the relative radius of both stars and therefore correlates with the orbital separation $a,\left(p_{\text {ecl }}=\frac{r_{1}+r_{2}}{a}\right)$. The separation $a$ can be calculated from the masses and the period $\left(a=\left(G \frac{m_{1}+m_{2}}{4 \pi^{2}}\right)^{1 / 3} \cdot P^{2 / 3}\right)$. We assumed for the eclipsing probability the median masses and radii of the published HW Vir systems (see Schaffenroth et al. 2018). The corrected period distribution can be found in Fig. 13. Up to a period of $0.35 \mathrm{~d}$ it appears to be a fairly flat distribution. For longer periods - up to one day - the number of systems drops significantly. Above $0.55 \mathrm{~d}$ we found only eight systems. This small number of systems does not allow significant conclusions to be drawn on the number of systems for periods longer than $13 \mathrm{~h}$.

\subsection{Selection effects}

For interpretation of the period distribution it is important to understand the selection effects that limit the detection of HW Vir systems at certain periods. To detect the shortest-period systems, a short cadence of observation of the light curve is essential. The light curve surveys we used have relatively random cadence. The shortest-period eclipsing system released by the

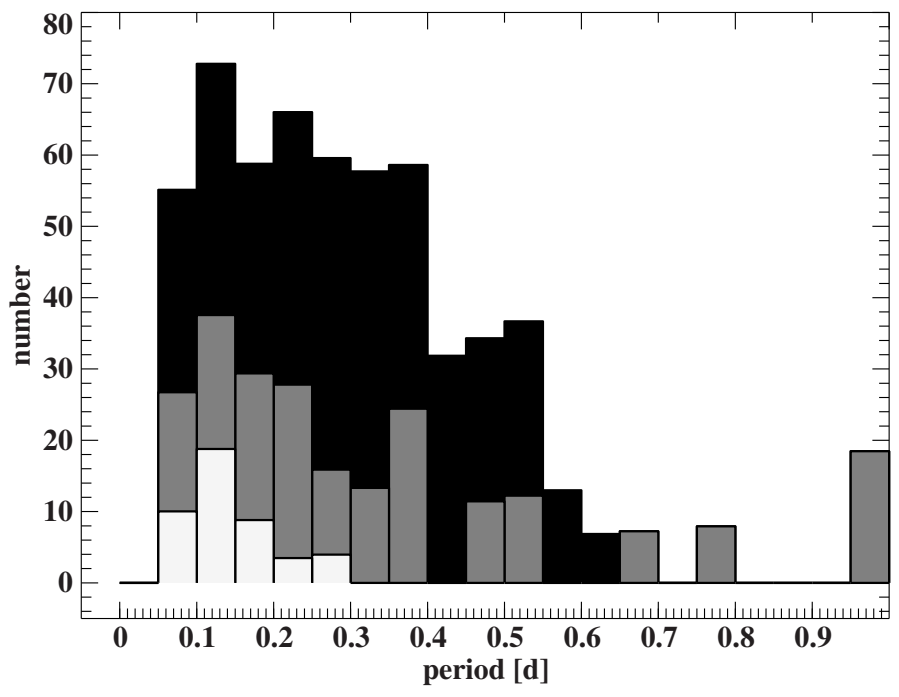

Fig. 13. Period distribution for all our targets corrected for the eclipsing probability. The currently published HW Vir systems are shown in white, the ATLAS targets in gray, and the OGLE targets in black.

OGLE team has a period of $0.052 \mathrm{~d}$ and is part of our target sample. The shortest-period system we found in the ATLAS survey has a period of $0.062 \mathrm{~d}$. In OGLE, three systems with significantly shorter periods have been found. We were able to find systems with shorter periods of around $0.05 \mathrm{~d}$ when phasing the ATLAS light curves of the sdB candidates from the hot subdwarf Gaia catalog (Geier et al. 2019). The question is, why did we not find such short-period systems in ATLAS? One explanation is that they might be very rare. From the OGLE catalog it is hard to say what the minimum period of a HW Vir system is, as there were no shorter-period systems released. To solve the question about the minimum period, we have to wait for photometric surveys observing a large number of systems with a better cadence and more epochs.

The eclipsing probability decreases substantially as the period increases, so it is not surprising that we do not find systems with periods longer than about a day. The eclipsing probability for those systems is less than $10 \%$. The reflection effect also gets weaker with increasing period. We expect to find only systems with an extremely high reflection effect for periods larger than one day assuming the quality of the light curves from the OGLE and ATLAS surveys. Such systems are much rarer than the typical systems (see the light curves of our targets shown in Figs. A.1 and A.2). To find longer-period sdB binaries with cool companions we need a larger sample and have to include the systems showing only the reflection effect, as well as observations with very accurate light curves.

\section{Discussion}

For population synthesis, the criteria for the ejection of the common envelope are crucially determined by the orbital-period distribution of post-CE binaries (Han et al. 2002). Hence, a period distribution as complete as possible is important for the understanding of those parameters.

\subsection{Companion masses and periods of the known reflection-effect binaries}

Figure 14 shows the companion masses of the published HW Vir systems (Schaffenroth et al. 2018, and references therein for 


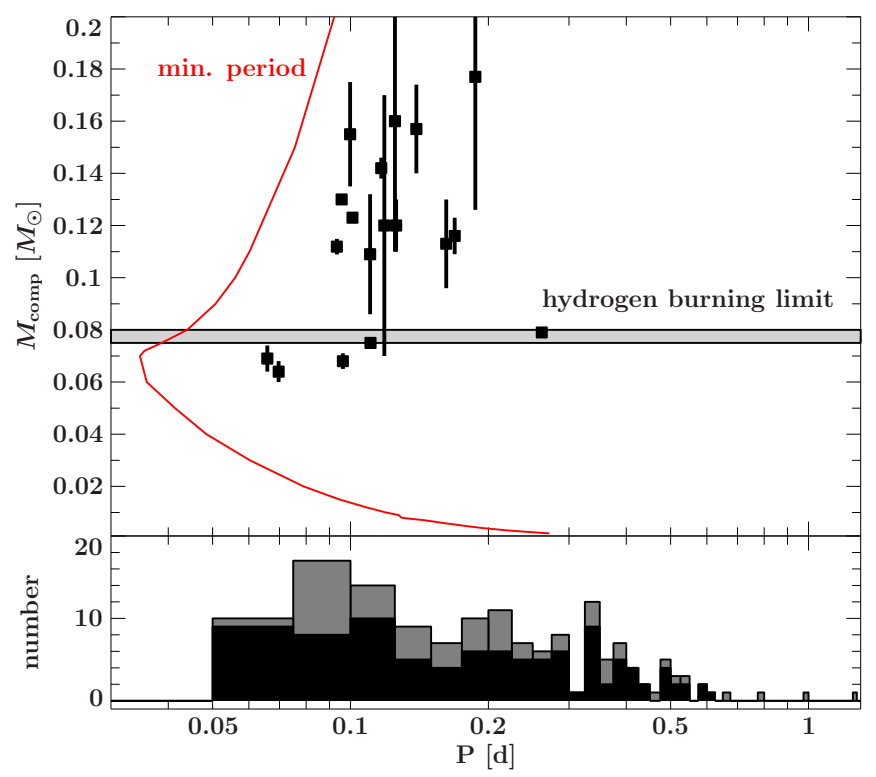

Fig. 14. Period/companion mass diagram of the published HW Vir systems. A summary of the parameters can be found in Schaffenroth et al (2018) and references therein. The gray area marks the hydrogenburning limit. Companions with smaller masses are substellar. The solid red line denotes the minimum period at which a companion of a certain mass can exist in orbit around a canonical-mass sdB, assuming the companion cannot exceed its Roche radius. In the lower panel the period distribution of the newly discovered HW Vir systems presented here is shown for comparison (OGLE targets in black and ATLAS targets in gray).

a summary of the orbital parameters) plotted against their orbital periods - there is no obvious correlation between companion mass and period. However, the confirmed substellar companions seem to be found preferentially in the shortest-period systems. The minimum period possible for a system consisting of an $\mathrm{sdB}$ and a companion of a certain mass can be calculated by assuming that the radius of the companion cannot exceed its Roche radius. To derive the Roche radius we used the formula by Eggleton (1983), which depends on the mass ratio and separation of the binary. For the radius of the companion we used the mass-radius relation by Baraffe et al. (1998), and for the sdB mass we adopt the canonical value $\left(\sim 0.47 M_{\odot}\right)$.

The minimum orbital period is reached for an $\mathrm{sdB}$ with a cool, low-mass companion in the brown dwarf mass range. When we look at the period distribution of the newly discovered systems, which is shown in Fig. 14 for comparison, we can see that the number of short-period systems below $0.1 \mathrm{~d}$ has increased substantially. In particular, in the period range below $0.1 \mathrm{~d}$, where all the currently confirmed brown dwarf companions have been found, we discovered 30 new systems, down to almost the minimal possible period at $0.04 \mathrm{~d}$ (see also Nelson et al. 2018).

To find possible Jupiter-mass planets we have to search for longer periods, because these planets get destroyed during the common-envelope phase if they are too close to the star. We estimate that they can only survive at periods longer than $0.2-0.25 \mathrm{~d}$ (see Fig. 14). The only eclipsing sdB binary with a cool, low-mass companion known to have such a period was AA Dor. The newly discovered systems increase the range of periods up to more than one day, allowing a search for close Jupiter-mass objects around hot subdwarf stars, which could be responsible for the formation of the sdB. This newly discovered sample provides a unique opportunity to study the

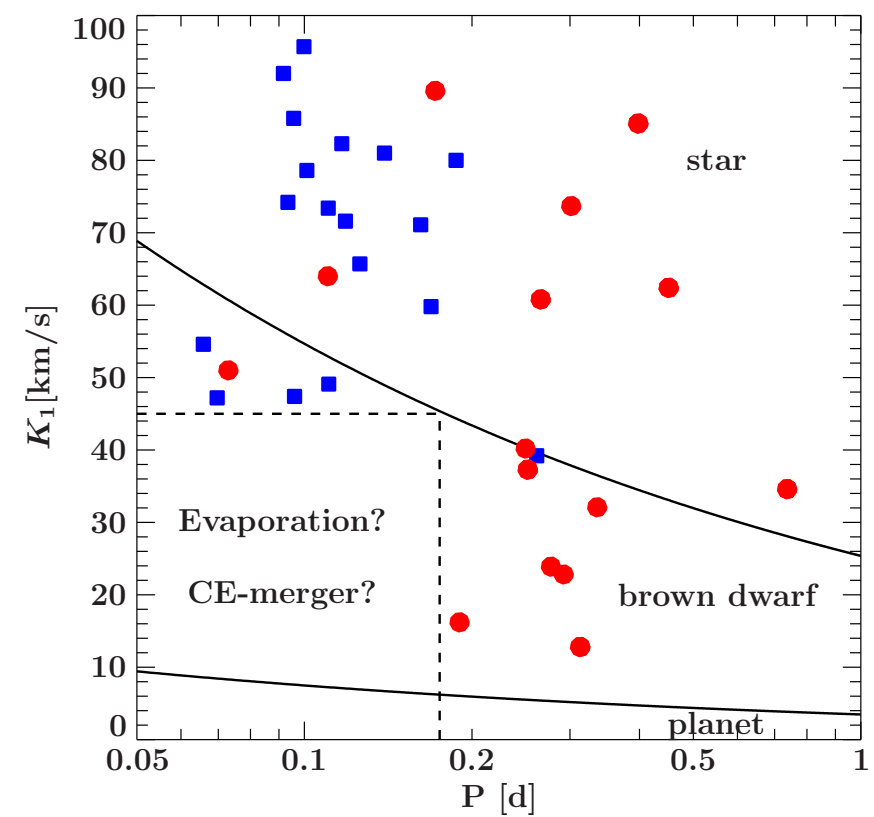

Fig. 15. Updated version of Fig. 4 from Schaffenroth et al. (2014b) adding the HW Vir systems published since then (see Schaffenroth et al. 2018, for a table of the parameters of all published HW Vir systems). Shown are the RV semi-amplitudes of all known sdB binaries with reflection effects and spectroscopic solutions plotted against their orbital periods. Blue squares mark eclipsing sdB binaries of HW Vir type where the companion mass is well constrained and red circles show systems without eclipses, where only lower limits can be derived for the companion masses. The dashed lines mark the regions to the right where the minimum companion masses derived from the binary mass function (assuming $0.47 M_{\odot}$ for the sdBs) exceed $0.01 M_{\odot}$ (lower curve) and $0.08 M_{\odot}$ (upper curve).

parameters of post-common-envelope systems over a large period range.

For noneclipsing systems, the absolute mass of the companion cannot be determined. Some first tests show, however, that the inclination and radii can be constrained with light curves of space-based quality (Schaffenroth et al., in prep.), which will allow us to constrain the companion masses. Assuming the canonical sdB mass, a minimum mass for all companions can be derived. An overview of the 33 known sdB binaries with reflection effects and known orbital parameters is shown in Fig. 15. Although only minimum masses can be derived for most of the companions, we can use this sample to do some statistics. While most companions are late M-dwarfs with masses close to $\sim 0.1 M_{\odot}$, there is no sharp drop below the hydrogen-burning limit. The fraction of close substellar companions is substantial. An obvious feature in Fig. 15 is the lack of binaries with periods shorter than $\sim 0.18 \mathrm{~d}$ and $K<47 \mathrm{~km} \mathrm{~s}^{-1}$ corresponding to companion masses of less than $\sim 0.06 M_{\odot}$. This feature is not caused by selection effects: the comparable radii of giant planets and stars close to the hydrogen-burning limit means that their eclipse depths would be similar, and their shorter orbital periods would mean the reflection effect should be as strong as or stronger than that of typical HW Vir binaries. An explanation for the lack of objects at short periods could be that the companion triggered the ejection of the envelope, but was destroyed during the common-envelope phase. This could also explain the formation of some of the single sdBs. Our target sample is ideal for studying this region in the diagram more thoroughly, as it significantly increases the number of known systems with periods smaller than $0.15 \mathrm{~d}$. 

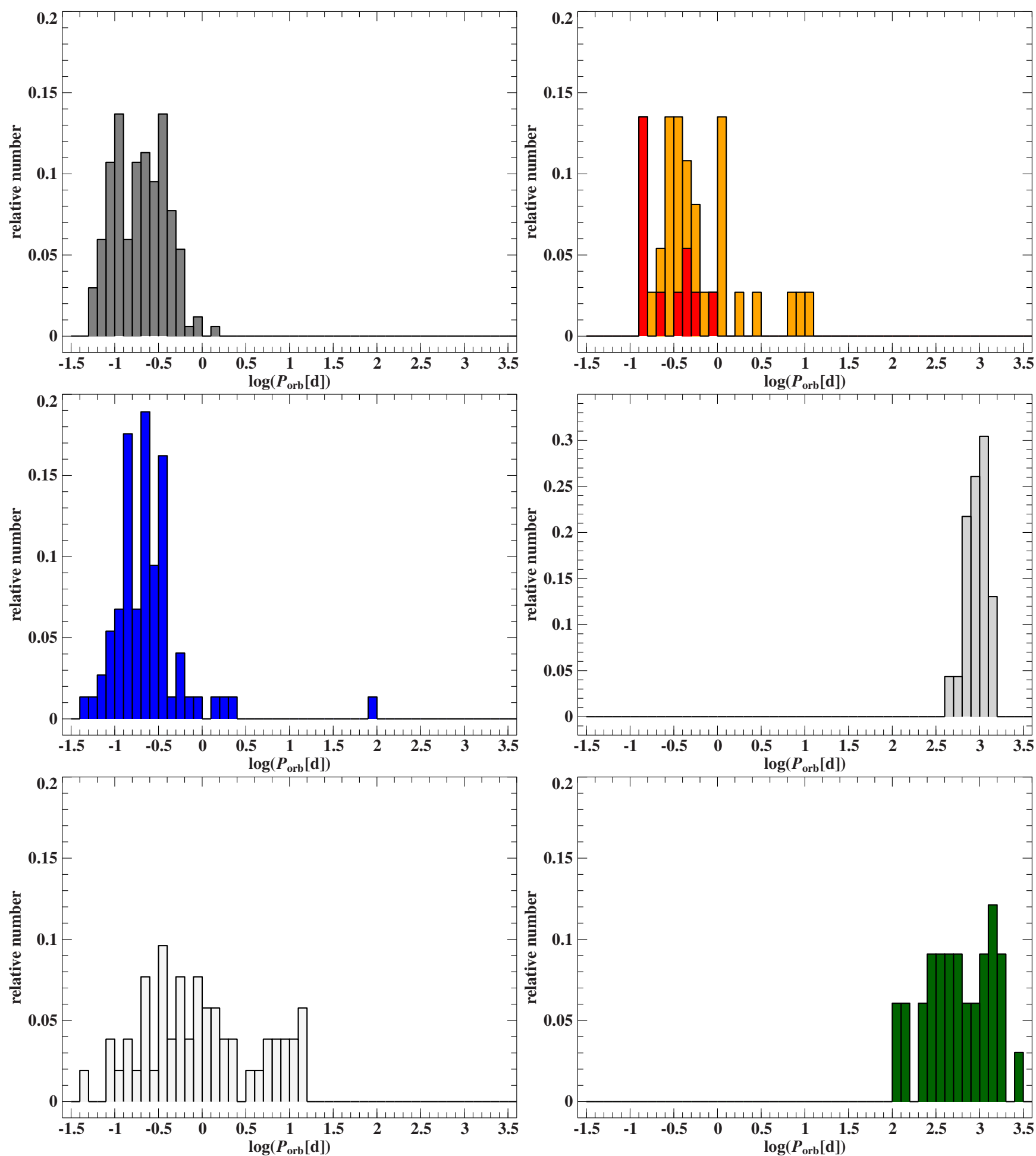

Fig. 16. Period distribution of different kinds of post-common-envelope systems. Left: top: all HW Vir systems including the HW Vir candidate systems from this paper (gray); middle: known eclipsing WD+dM/BD systems from Parsons et al. (2015) (blue); bottom: known sdB+WD systems from Kupfer et al. (2015) (white). Right: top: known eclipsing binary central stars of planetary nebula showing a reflection effect (red) and noneclipsing binary central stars of planetary nebula showing a reflection effect (orange ${ }^{3}$ ); middle: known sdB + main sequence companions from the Roche lobe overflow channel (Vos et al. 2019, light-gray); bottom: post-AGB binaries from Oomen et al. (2018, dark green).

\subsection{Comparison with related (eclipsing) binary populations}

It is also interesting to compare our period distribution with the distribution of other types of post-common envelope systems. This is shown in Fig. 16. The eclipsing WD+dM/BD systems show a very similar distribution. However, the longest-period systems have periods of $2.3 \mathrm{~d}$. All the systems with periods longer than $0.5 \mathrm{~d}$ have WD primaries with masses greater than $0.55 M_{\odot}$ and have to be post-AGB stars. The longest-period system KOI-3278 with a period of $88 \mathrm{~d}$ is a post-common envelope system of a CO-WD with a $\mathrm{G}$ type companion (Parsons et al. 2015). It is the longest-period eclipsing post-common-envelope 
system known. As the primary is a $\mathrm{CO}$ white dwarf it has to be a post-AGB binary. The white dwarf is much smaller than the subdwarf, which means that the eclipsing probability is much smaller for white dwarf binaries. However, they are much more common than hot subdwarf stars.

As already discussed in Kupfer et al. (2015) the period distribution of the known sdB+WD systems resulting from the second common envelope channel is much broader than the period distribution of the reflection-effect systems with a secondary peak at periods of several days.

Another type of post-common-envelope systems are the binary central stars of planetary nebula. As only 11 eclipsing systems are known, we also added systems which only show the reflection effect. The period distribution of the eclipsing bCSPNs with cool companions looks very similar to the distribution of the HW Vir systems. However, systems showing only the reflection effect and no eclipses are found up to periods of several days, almost as long as the $\mathrm{sdB}+\mathrm{WD}$ systems. The primary can be a very hot WD or sdO and so the amplitude of the reflection effect is relatively large in some but not all cases. The bCSPNs can either be post-RGB or post-AGB systems.

The longest-period post-common-envelope system with an sdB primary has a period of $27.8 \mathrm{~d}$, but the nature of the companion has not yet been determined. For comparison we also added the group of long-period sdB systems with FGK companions showing composite spectra. They are found at periods of a few $100 \mathrm{~d}$ which means they were formed through the Roche lobe overflow channel. The shortest period for such $\mathrm{sdB}$ binaries is $479 \mathrm{~d}$ (Vos et al. 2019). No systems with periods in the range $\sim 28-480 \mathrm{~d}$ have been found yet.

Oomen et al. (2018) published a sample of 33 post-AGB binaries. They have periods similar to those of long-period sdB binaries and share other characteristics with this latter group (e.g., significant eccentricities in many systems); population synthesis, on the other hand, predicted periods of a few days for common-envelope systems (Nie et al. 2012). Oomen et al. (2018) claim that post-AGB stars with periods less than $100 \mathrm{~d}$ should fill their Roche lobes and therefore evolve into bCSPNs quickly or contain hotter post-AGB primaries. Those should be distinguishable from post-RGB stars quite easily, as they are expected to be much more luminous.

\section{Summary and outlook}

In the EREBOS project we study a large sample of homogeneously selected HW Vir systems. We investigated two photometric surveys to find more such systems and increased the number of known systems by a factor of almost ten. We plan a photometric and spectroscopic follow-up of as many targets as possible to determine the fundamental stellar $(M, R)$, atmospheric $\left(T_{\text {eff }}, \log g\right)$, and binary parameters $(a, P)$. At the moment we already have spectroscopic follow-up for 28 objects. For several of our systems we took photometric follow-up in several bands $(B, V, R)$, which is essential for modeling the reflection effect.

For 25 of our targets, it has been spectroscopically confirmed that they are indeed systems consisting of a hot subdwarf primary and a cool, low-mass companion; only two targets have a DA primary. Four systems in our sample were confirmed to be central stars of planetary nebula. This means that $\sim 90 \%$ of our target sample are most likely to be eclipsing hot subdwarf binaries.

The main goal of EREBOS is to investigate HW Vir systems over the whole range of the period distribution. We hope to improve the understanding of the common-envelope phase by investigating a large number of post-common-envelope binaries. Moreover, we are especially interested in the influence of the lowest-mass companions - brown dwarfs or massive planets on stellar evolution. A future goal is an improved physical model of the reflection effect, which we hope to achieve with this huge sample of reflection-effect binaries.

A byproduct that will emerge from our sample is a massradius relation for cool, low-mass objects which are highly irradiated by a hot companion. This should shed light on the question of how much such objects are inflated.

The TESS mission, which is observing at the moment, provides $27 \mathrm{~d}$ light curves of each field and, for a few bright targets, light curves with a 2 min cadence are transmitted. The fullframe images are transferred every $30 \mathrm{~min}$, allowing us to derive the light curves of all targets. This will give $27 \mathrm{~d}$ light curves of space-based quality, allowing us to detect reflection effects with periods of several days and to find the longest-period reflectioneffect systems. It will also provide excellent light curves to expand our target sample further. Additionally, several groundbased surveys are providing or will provide excellent light-curve data in several photometric bands, which will find a large number of new HW Vir systems (e.g., ZTF and BlackGEM, of which we are now members).

Acknowledgements. Based on observations collected at the European Organisation for Astronomical Research in the Southern Hemisphere under ESO programme(s) 092.D-0040(A), 095.D-0167(A), 196.D-0214(A-D), 099.D-0217(A), 0101.D-0791(A). Also based on observations obtained at the Southern Astrophysical Research (SOAR) telescope, which is a joint project of the Ministerio da Ciência, Tecnologia, Inovações e Comunicações (MCTIC) do Brasil, the US National Optical Astronomy Observatory (NOAO), the University of North Carolina at Chapel Hill (UNC), and Michigan State University (MSU). We would like to thank the Chilean Time Allocation Committee for awarding us time for complementing the EREBOS Project with the proposal CL-2016B-018. Unfortunately, there was an error on our side and the finder chart was wrongly marked so that the data were not used in the analysis presented in this paper. V.S. is supported by the Deutsche Forschungsgemeinschaft (DFG) through grant GE 2506/9-1. BB is supported by the National Science Foundation grant AST1812874. DK acknowledges financial support from the University of the Western Cape and the National Research Foundation of South Africa. IP acknowledges funding by the Deutsche Forschungsgemeinschaft under grant GE2506/12-1. This work was supported by the National Science Foundation through grant PHY 17-148958. V.S. and S.G. were supported by the DAAD PPP USA for this project. Thereby, we would also like to thank the Kavli Institute for Theoretical Physics, UC Santa Barbara for hosting V.S and S.G. during the stay funded by DAAD PPP USA, where a large part of the manuscript was written. This research has made use of ISIS functions (ISISscripts) provided by ECAP/Remeis observatory and MIT (http://www . sternwarte.uni-erlangen.de/isis/). This work has made use of data from the European Space Agency (ESA) mission Gaia (https://www.cosmos.esa.int/gaia), processed by the Gaia Data Processing and Analysis Consortium (DPAC, https://www.cosmos.esa.int/ web/gaia/dpac/consortium). Funding for the DPAC has been provided by national institutions, in particular the institutions participating in the Gaia Multilateral Agreement.

\section{References}

Afşar, M., \& Ibanoğlu, C. 2008, MNRAS, 391, 802

Almeida, L. A., Damineli, A., Rodrigues, C. V., Pereira, M. G., \& Jablonski, F. 2017, MNRAS, 472, 3093

Althaus, L. G., Miller Bertolami, M. M., \& Córsico, A. H. 2013, A\&A, 557, A19 Bailer-Jones, C. A. L., Rybizki, J., Fouesneau, M., Mantelet, G., \& Andrae, R. 2018, VizieR Online Data Catalog: I/347

Baraffe, I., Chabrier, G., Allard, F., \& Hauschildt, P. H. 1998, A\&A, 337, 403

Budaj, J. 2011, AJ, 141, 59

Clemens, J. C., Crain, J. A., \& Anderson, R. 2004, in Ground-based Instrumentation for Astronomy, eds. A. F. M. Moorwood, \& M. Iye, Proc. SPIE, 5492, 331

Dorman, B., Rood, R. T., \& O'Connell, R. W. 1993, APJ, 419, 596

Eggleton, P. P. 1983, ApJ, 268, 368

Gaia Collaboration (Babusiaux, C., et al.) 2018a, A\&A , 616, A10 
Gaia Collaboration (Brown, A. G. A., et al.) 2018b, A\&A, 616, A1

Geier, S., Schaffenroth, V., Drechsel, H., et al. 2011, APJ, 731, L22

Geier, S., Raddi, R., Gentile Fusillo, N. P., \& Marsh, T. R. 2019, A\&A, 621, A38

Gentile Fusillo, N. P., Gänsicke, B. T., \& Greiss, S. 2015, MNRAS, 448, 2260

Gentile Fusillo, N. P., Tremblay, P.-E., Gänsicke, B. T., et al. 2019, MNRAS, 482,4570

Han, Z., Podsiadlowski, P., Maxted, P. F. L., Marsh, T. R., \& Ivanova, N. 2002, MNRAS, 336, 449

Heber, U. 2009, ARA\&A, 47, 211

Heber, U. 2017, in Stripped Red Giants - Helium Core White Dwarf Progenitors and their sdB Siblings (San Francisco: ASP), ASP Conf. Ser., 509, 85

Heber, U., Reid, I. N., \& Werner, K. 2000, A\&A, 363, 198

Hillwig, T. C., Frew, D. J., Reindl, N., et al. 2017, AJ, 153, 24

Hirsch, H. 2009, Phd Thesis, Friedrich Alexander Universität Erlangen Nürnberg, Germany

Jeffery, C. S., \& Ramsay, G. 2014, MNRAS, 442, L61

Kupfer, T., Geier, S., Heber, U., et al. 2015, A\&A, 576, A44

Lallement, R., Vergely, J.-L., Valette, B., et al. 2014, A\&A, 561, A91

Lomb, N. R. 1976, Ap\&SS, 39, 447

Maxted, P. F. L., Heber, U., Marsh, T. R., \& North, R. C. 2001, MNRAS, 326, 1391

Miller Bertolami, M. M. 2016, A\&A, 588, A25

Miszalski, B., Acker, A., Moffat, A. F. J., Parker, Q. A., \& Udalski, A. 2009, VizieR Online Data Catalog: 349

Nelson, L., Schwab, J., Ristic, M., \& Rappaport, S. 2018, ApJ, 866, 88
Nie, J. D., Wood, P. R., \& Nicholls, C. P. 2012, MNRAS, 423, 2764 Oomen, G.-M., Van Winckel, H., Pols, O., et al. 2018, A\&A, 620, A85 Parsons, S. G., Marsh, T. R., Copperwheat, C. M., et al. 2010, MNRAS, 402, 2591

Parsons, S. G., Agurto-Gangas, C., Gänsicke, B. T., et al. 2015, MNRAS, 449, 2194

Pietrukowicz, P., Mróz, P., Soszyński, I., et al. 2013, Acta Astron., 63, 115

Riello, M., De Angeli, F., Evans, D. W., et al. 2018, A\&A, 616, A3

Scargle, J. D. 1982, ApJ, 263, 835

Schaffenroth, V., Geier, S., Drechsel, H., et al. 2013, A\&A, 553, A18

Schaffenroth, V., Geier, S., Heber, U., et al. 2014a, A\&A, 564, A98

Schaffenroth, V., Classen, L., Nagel, K., et al. 2014b, A\&A, 570, A70

Schaffenroth, V., Barlow, B. N., Drechsel, H., \& Dunlap, B. H. 2015, A\&A, 576, A123

Schaffenroth, V., Geier, S., Heber, U., et al. 2018, A\&A, 614, A77

Soker, N. 1998, AJ, 116, 1308

Soszyński, I., Stępień, K., Pilecki, B., et al. 2015, Acta Astron., 65, 39

Soszyński, I., Pawlak, M., Pietrukowicz, P., et al. 2016, Acta Astron., 66, 405

Tody, D. 1986, in Instrumentation in Astronomy VI, ed. D. L. Crawford, Proc SPIE, 627, 733

Tonry, J. L., Denneau, L., Heinze, A. N., et al. 2018, PASP, 130, 064505 Udalski, A., Pont, F., Naef, D., et al. 2008, A\&A, 482, 299

Udalski, A., Szymański, M. K., \& Szymański, G. 2015, Acta Astron., 65, 1

Vos, J., Vučković, M., Chen, X., et al. 2019, MNRAS, 482, 4592

Wilson, R. E. 1990, ApJ, 356, 613 


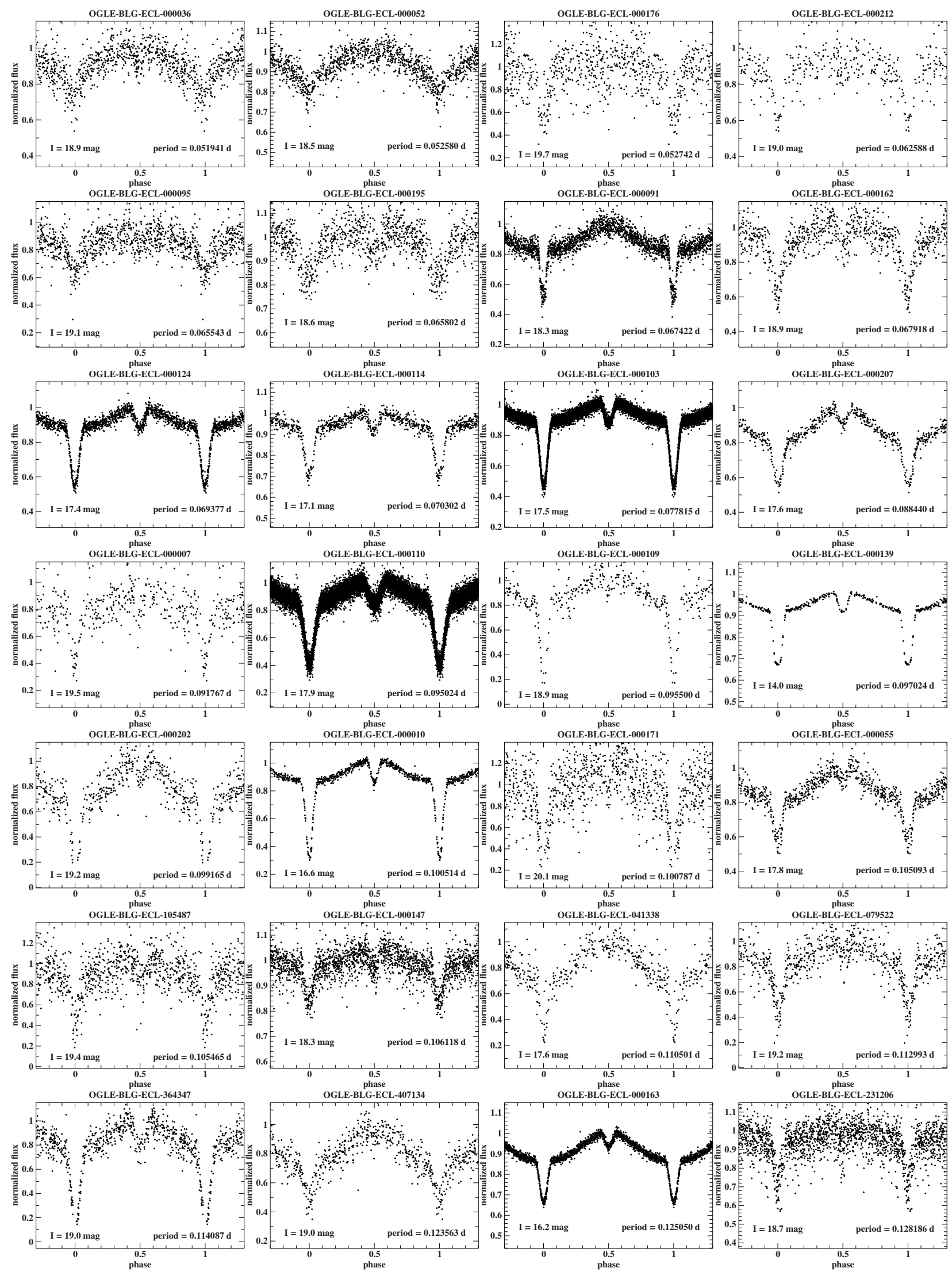

Fig. A.1. Phased light curves of all our HW Vir candidates from the OGLE survey. 

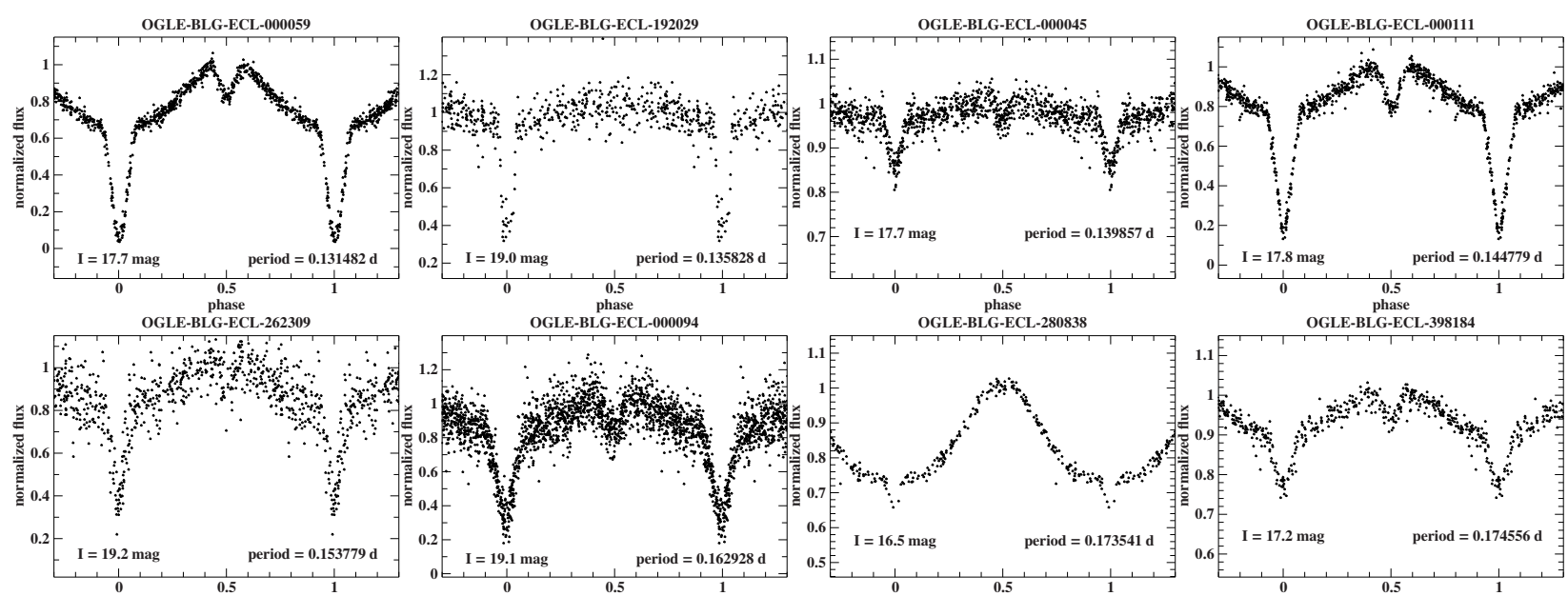

phase
OGLE-BLG-ECL-410872

phase
OGLE-BLG-ECL-318416

phase
OGLE-BLG-ECL-207454

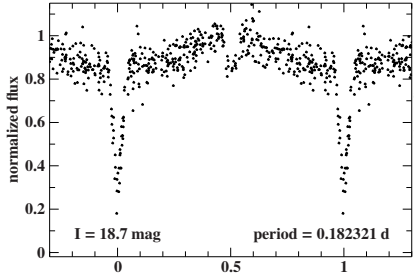

phase
OGLE-BLG-ECL-199157
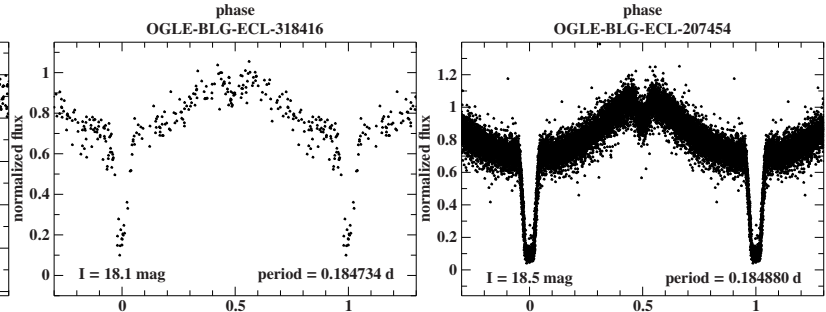

phase
OGLE-BLG-ECL-322700

phase
OGLE-BLG-ECL-123407
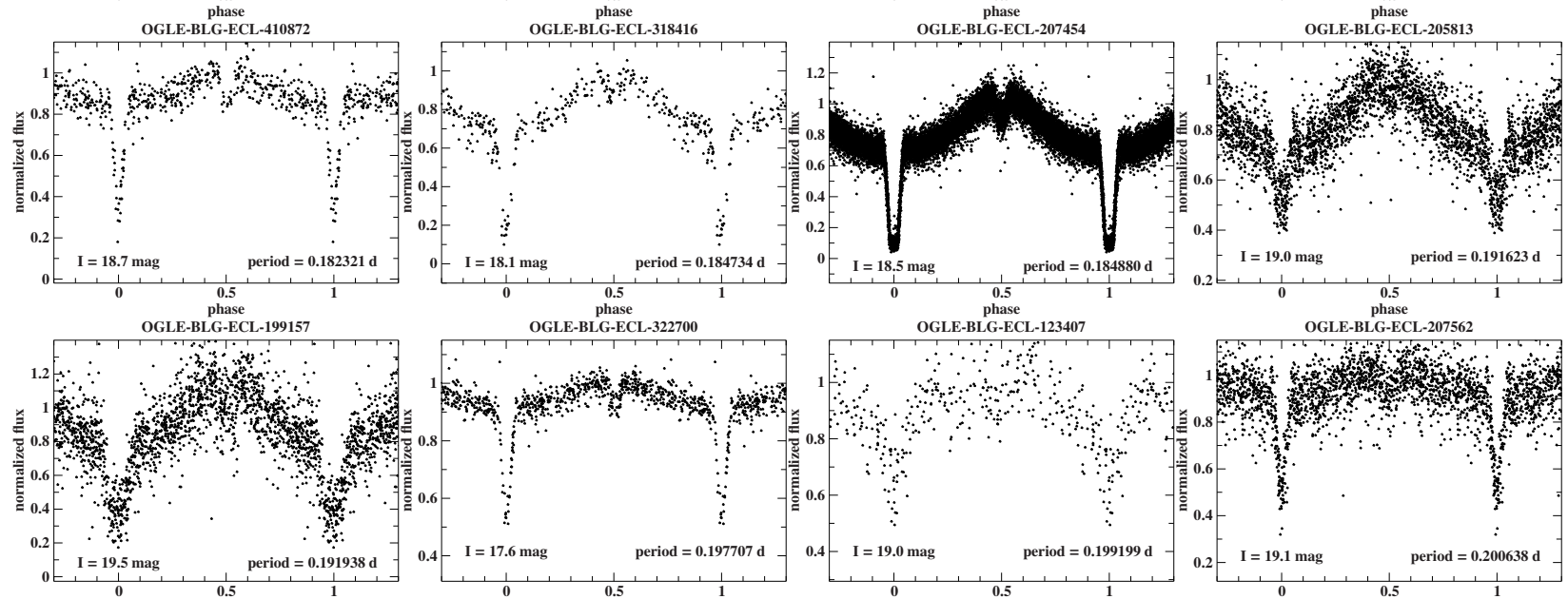

phase
OGLE-BLG-ECL-207562
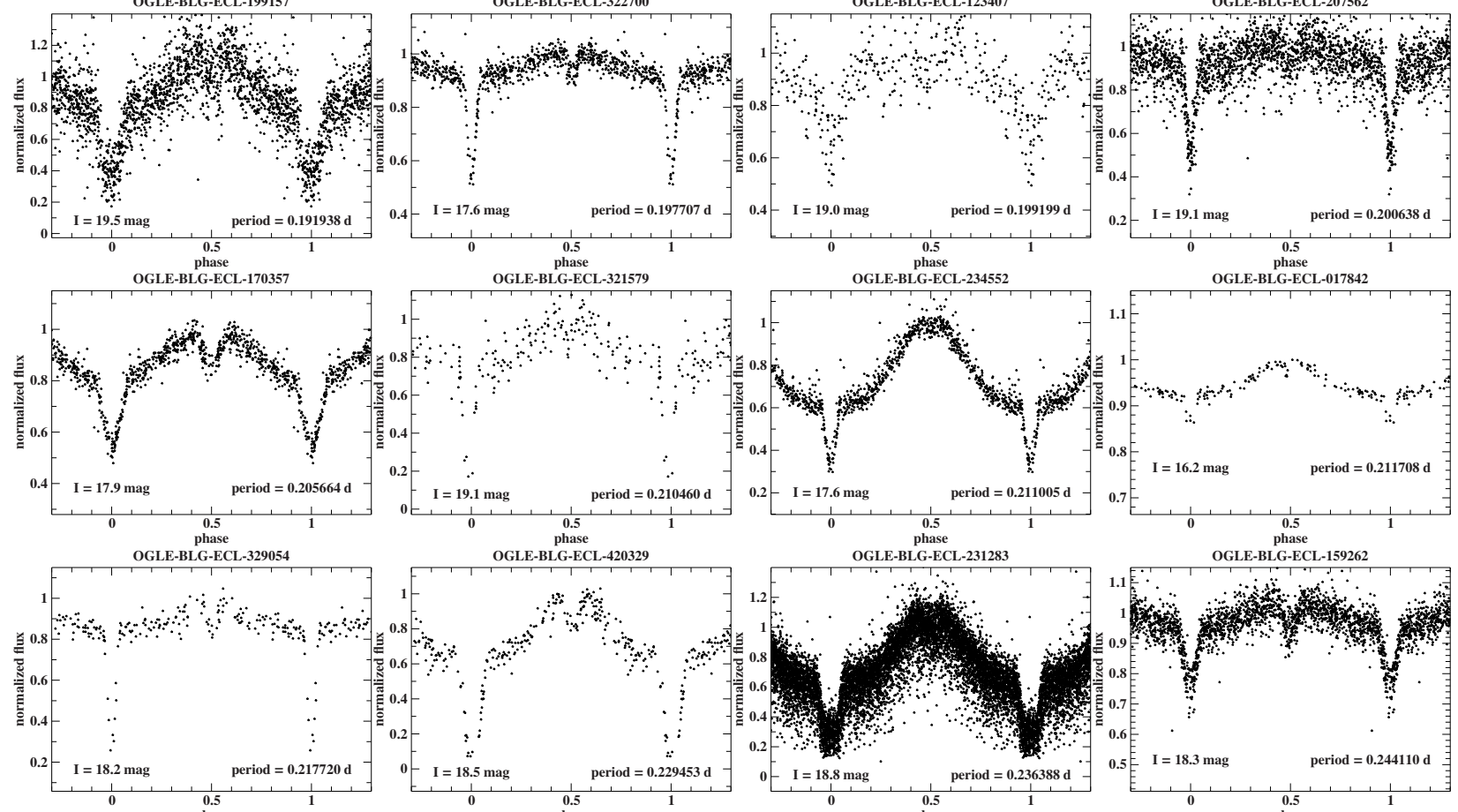

phase
OGLE-BLG-ECL-420329
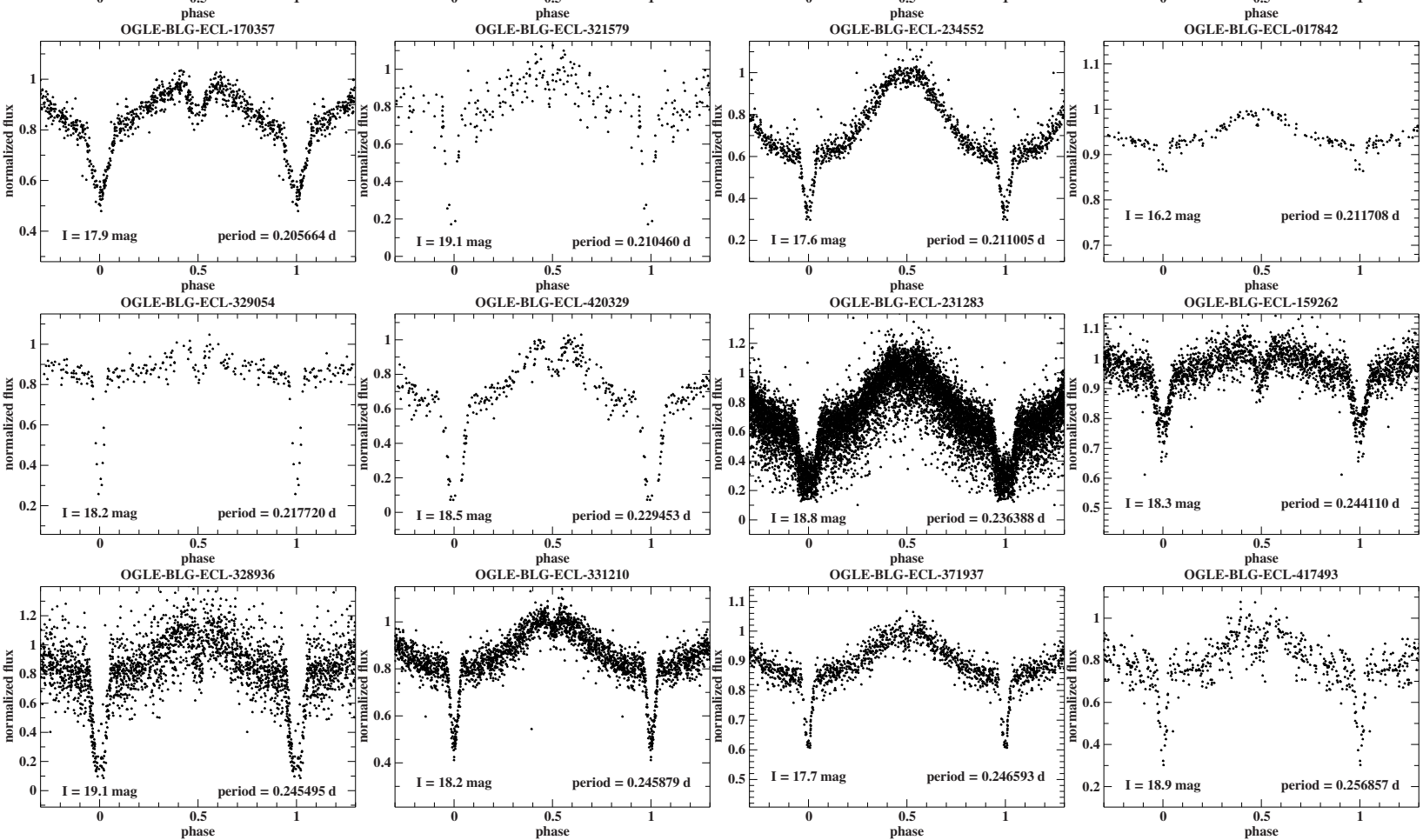

Fig. A.1. continued. 

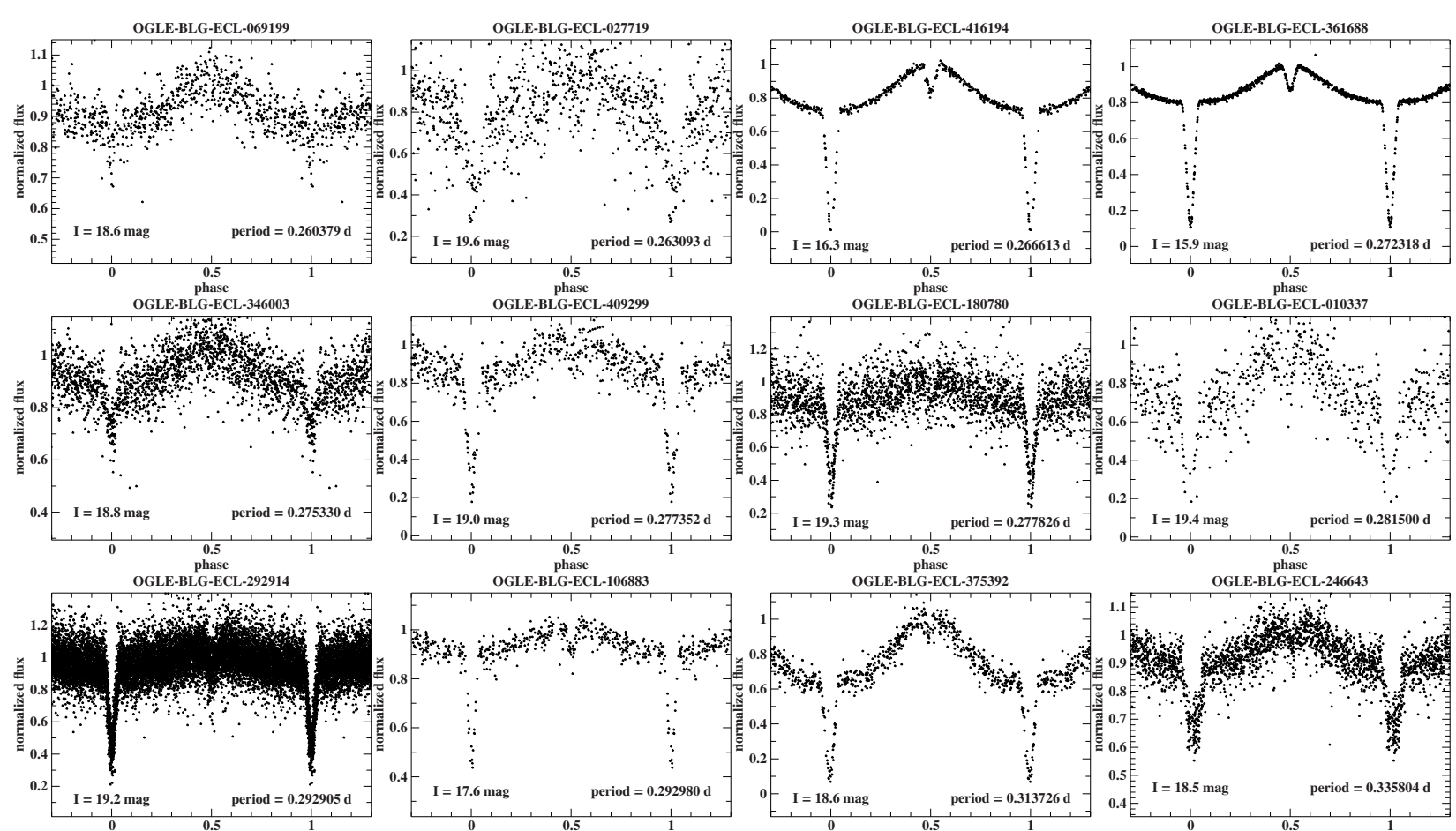

phase
OGLE-BLG-ECL-106883
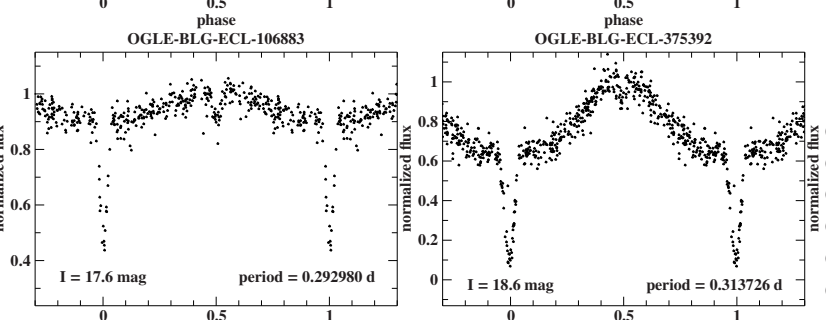

OGLE-BLGG-ECL-246643
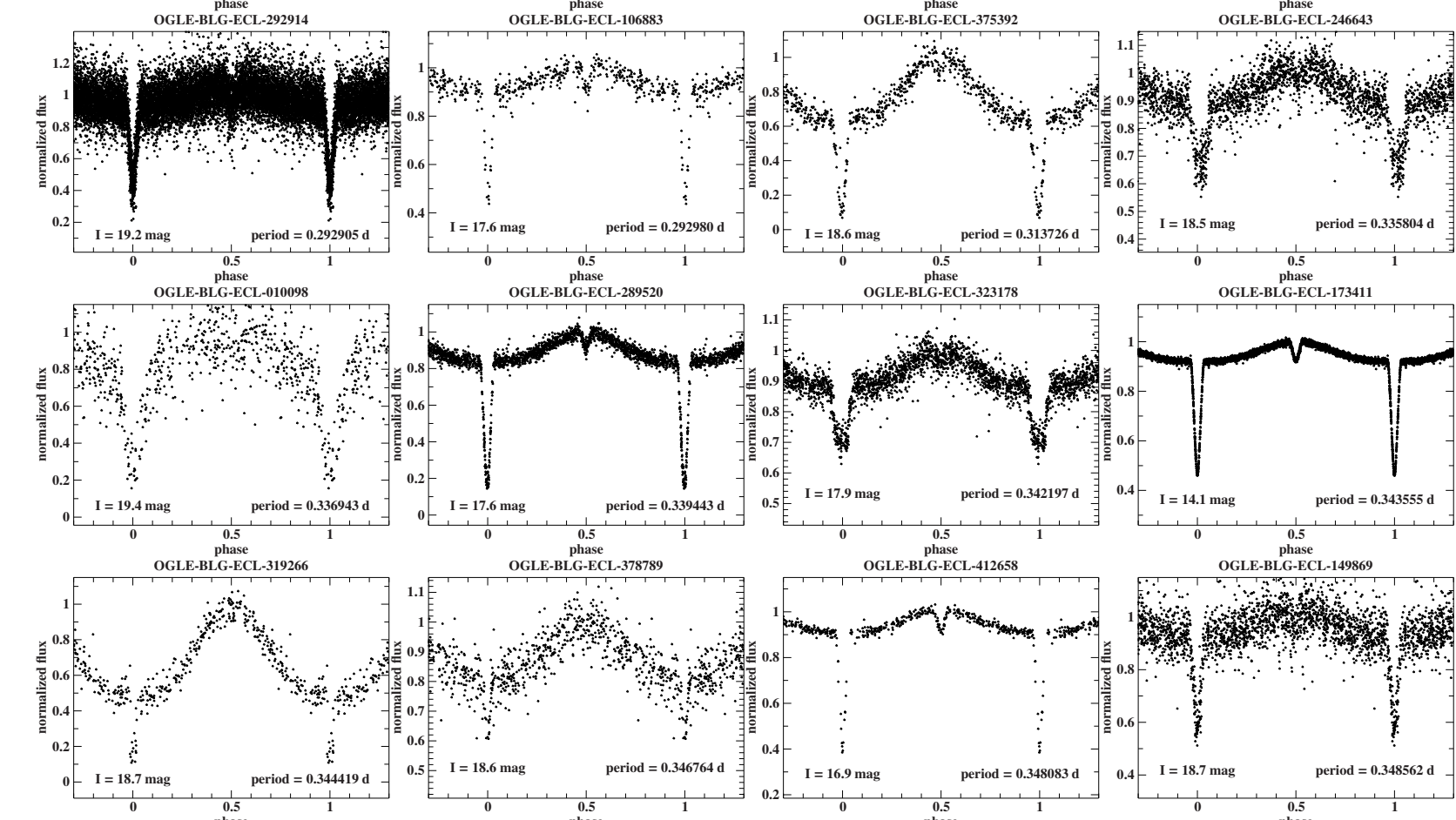

0.5
phase
OGLE-BLG-ECL-378789
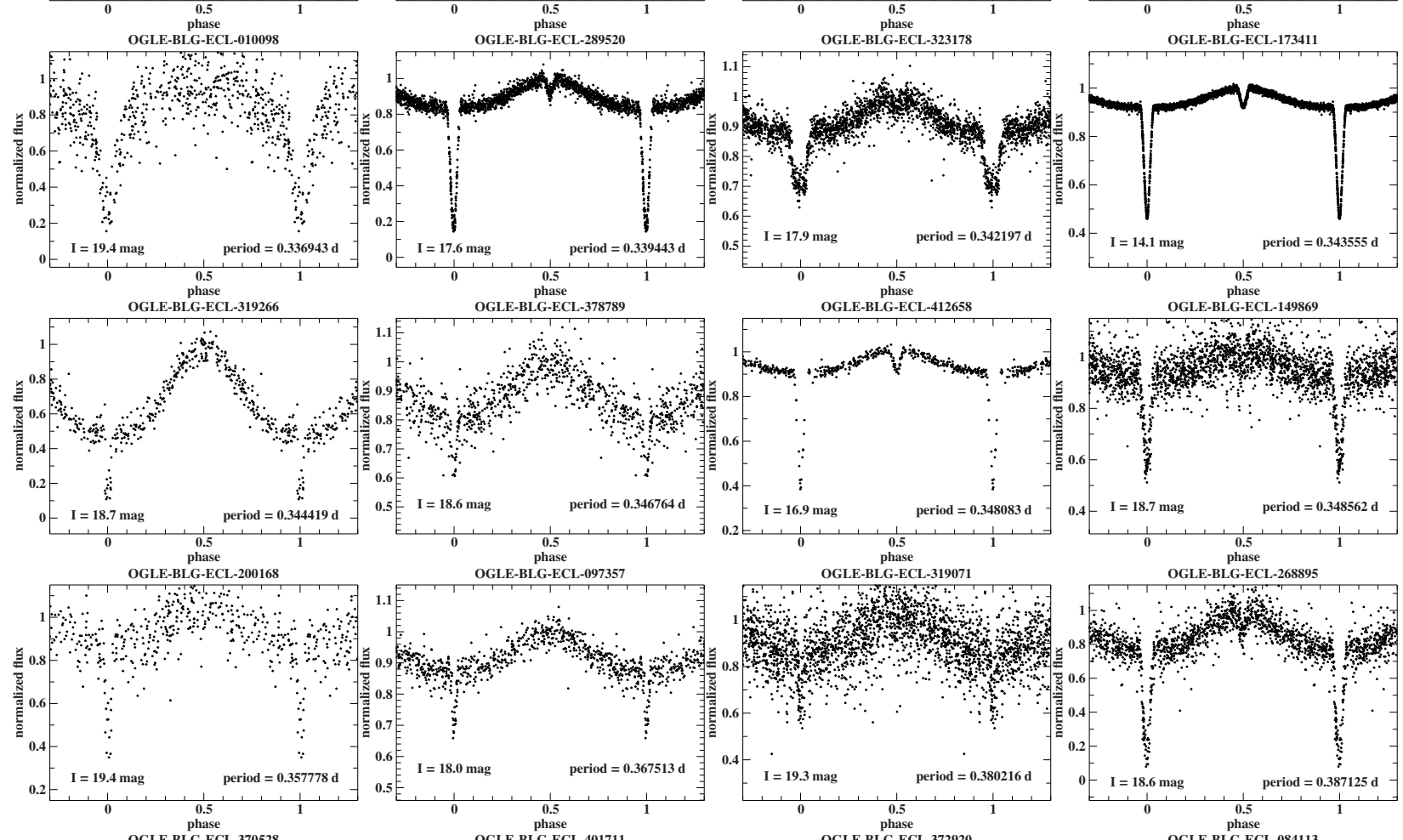

phase
OGLE-BLG-ECL-319071

phase
OGLE-BLG-ECL-268895
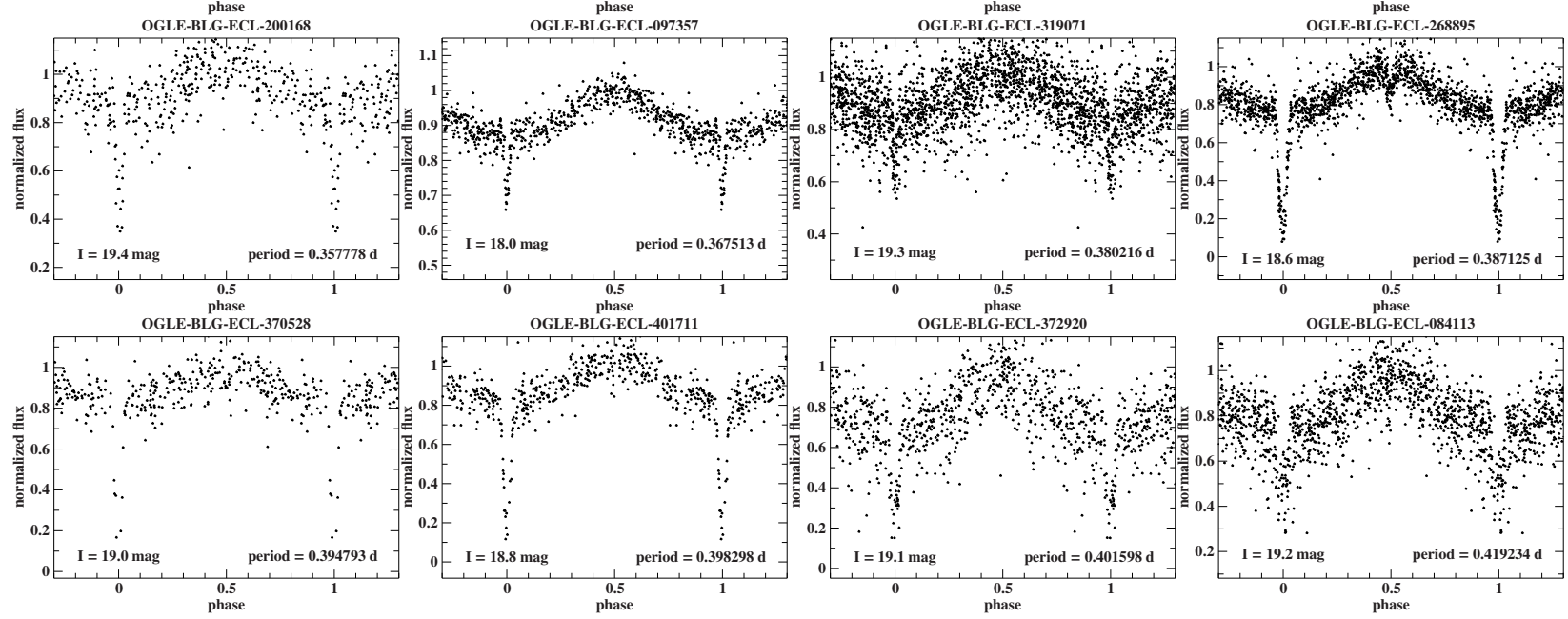

Fig. A.1. continued. 


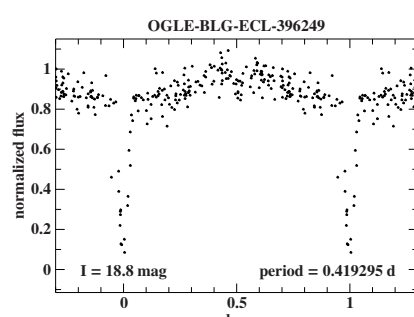

phase
OGLE-BLG-ECL-309483

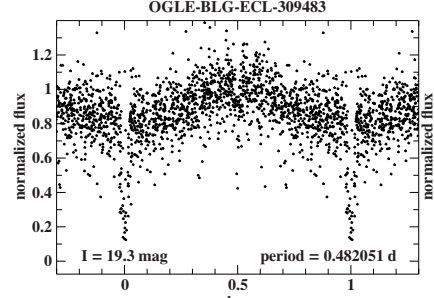

OGLE-BLG-ECL-360680
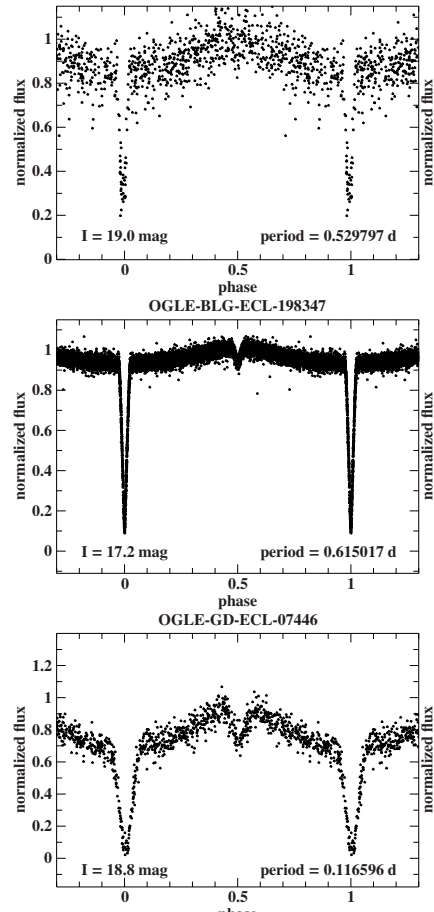

phase
OGLE-GD-ECL-00586

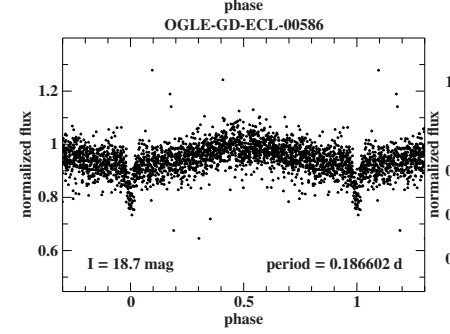

Fig. A.1. continued.

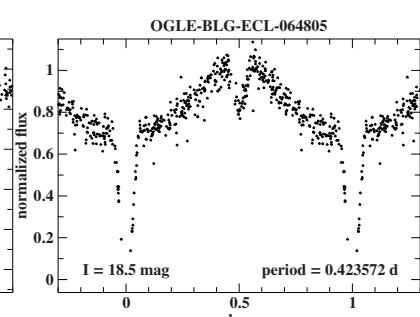

phase
OGLE-BLG-ECL-343208

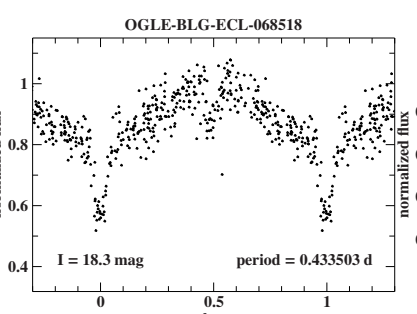

13 की

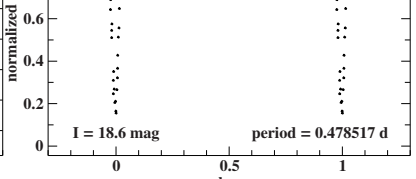

OGLE-BLG-ECL-121084

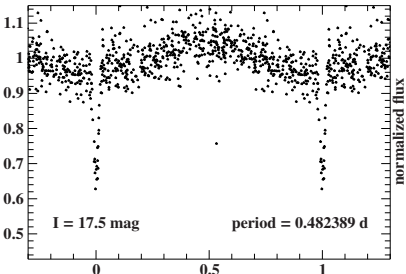

phase
OGLE-BLG-ECL-370434
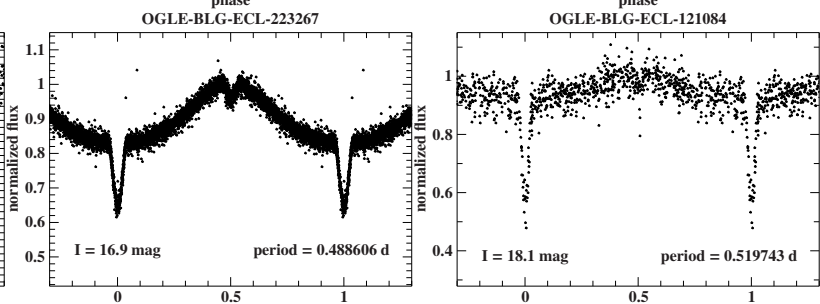

phase
OGLE-BLG-ECL-373886
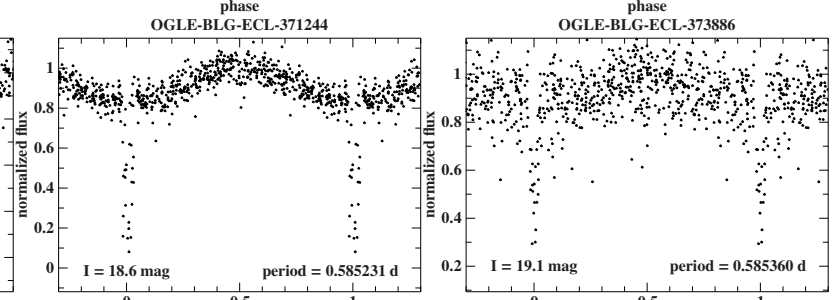

मnस
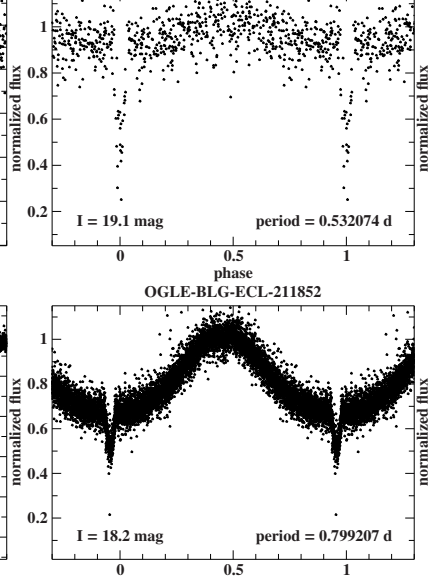

phase
OGLE-GD-ECL-10384

phase
OGLE-GD-ECL-09869
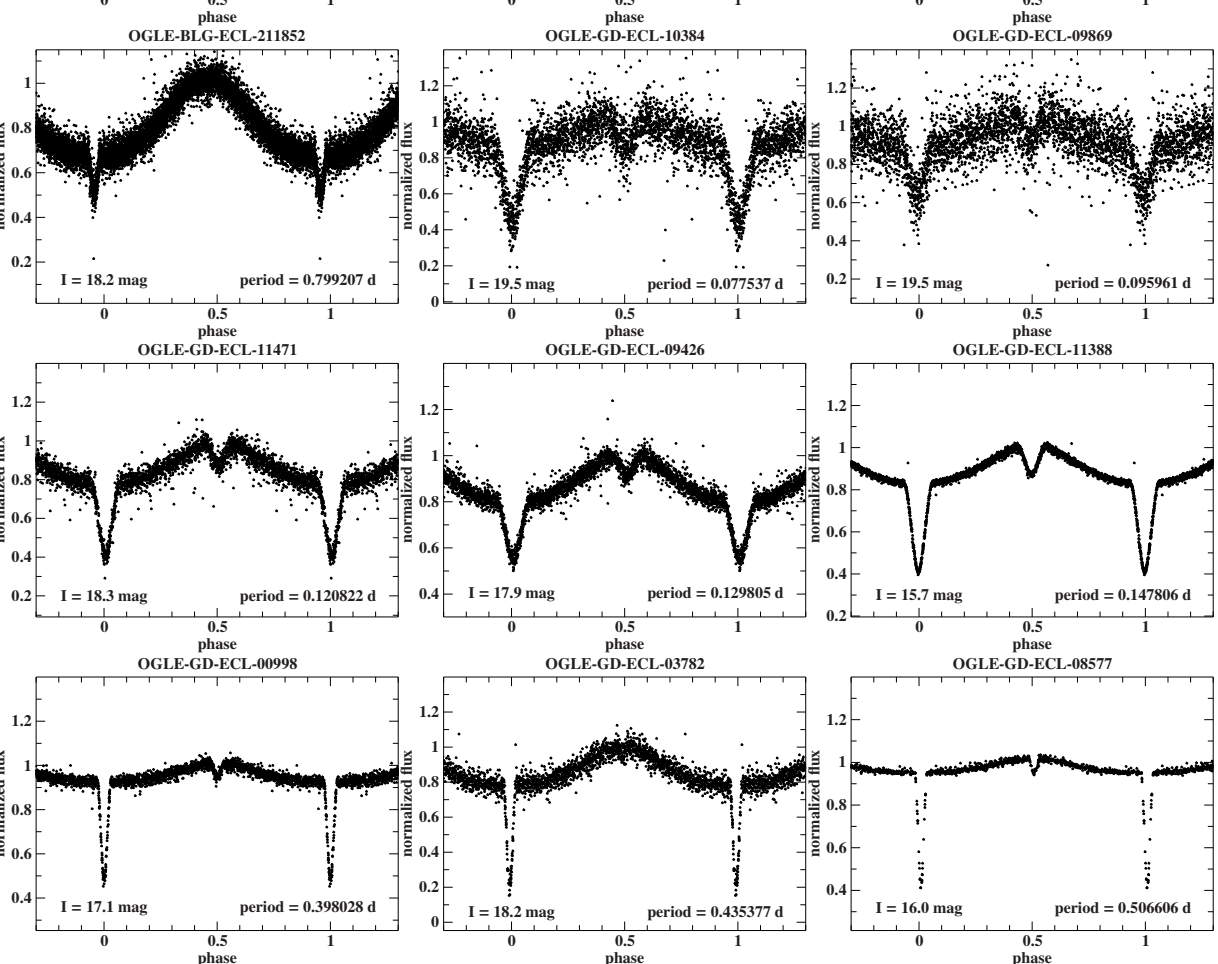
V. Schaffenroth et al.: The EREBOS project
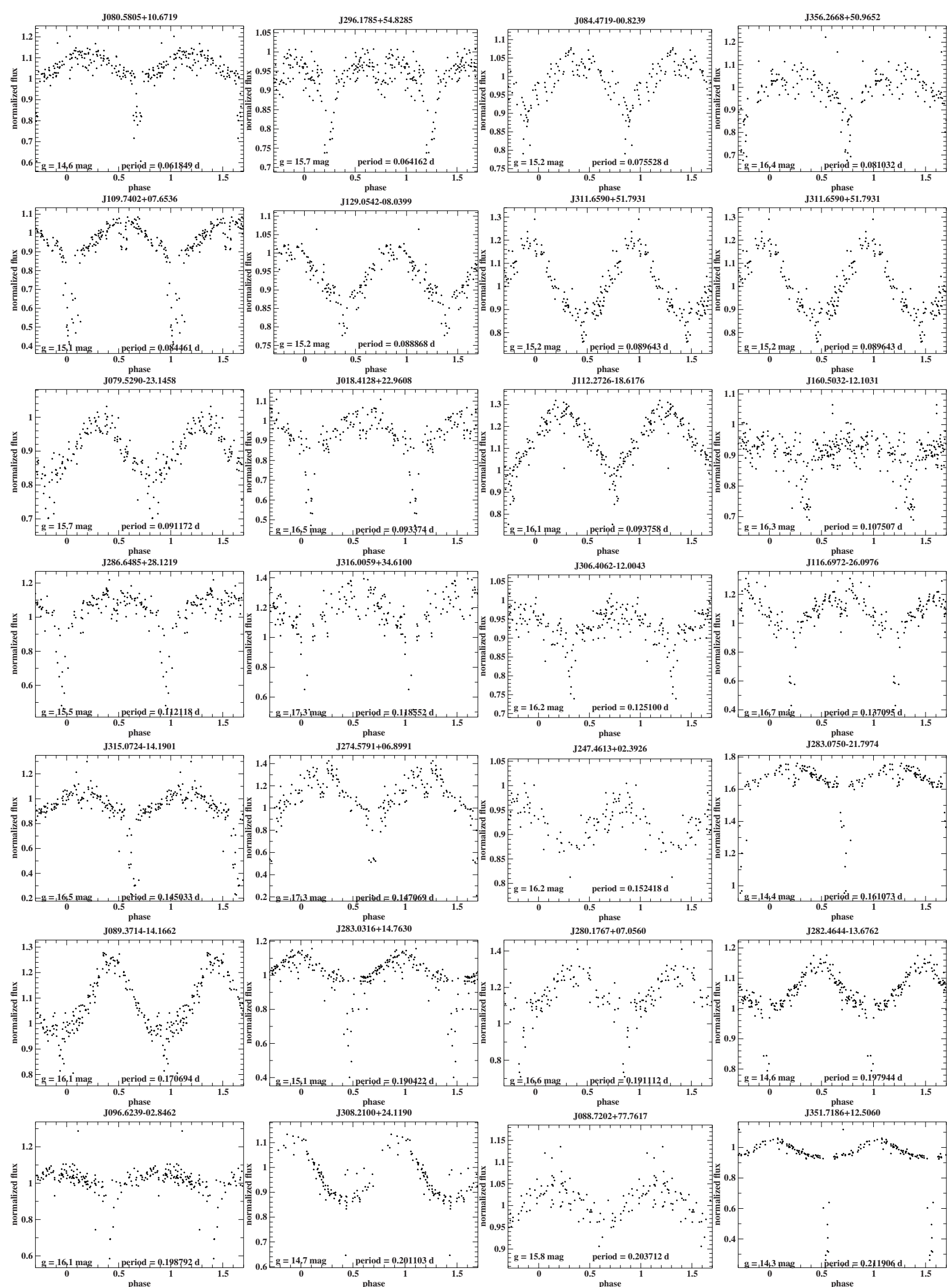

Fig. A.2. Phased light curves of all our HW Vir candidates from the ATLAS survey. 
A\&A 630, A80 (2019)

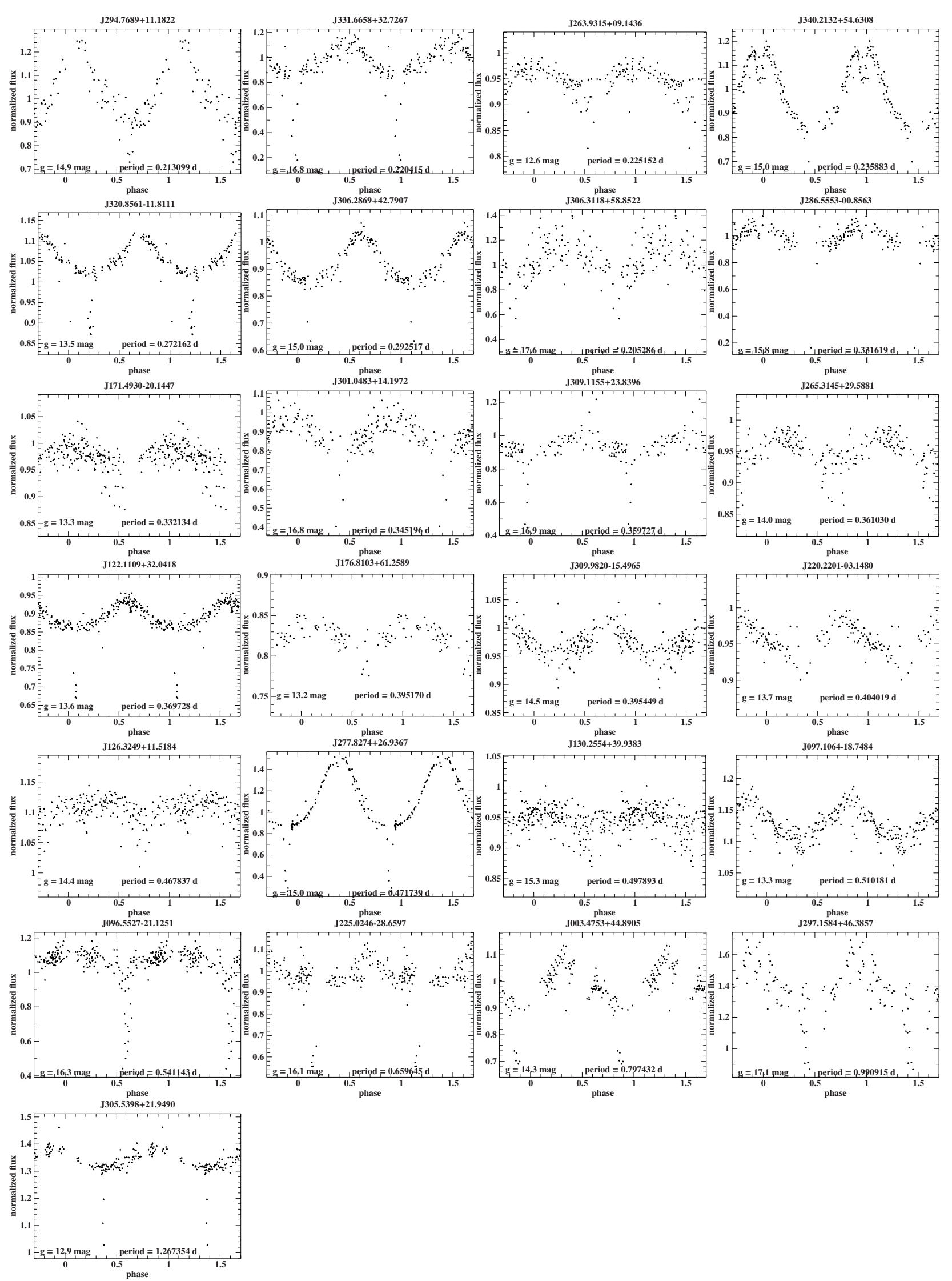

Fig. A.2. continued. 


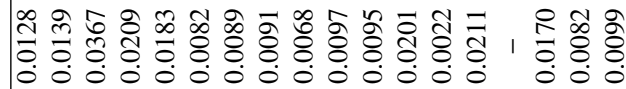

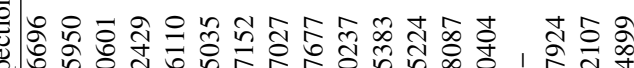

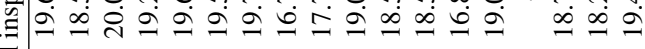

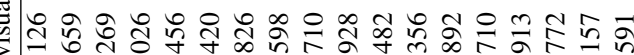

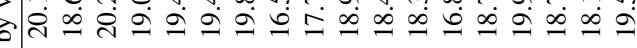

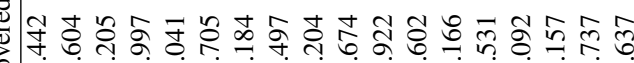

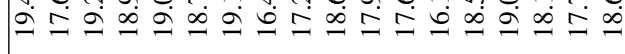

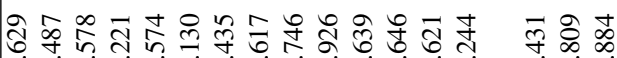
o के

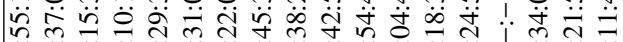

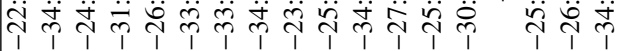

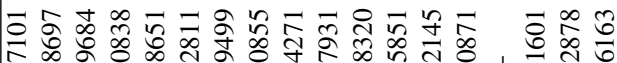
n்

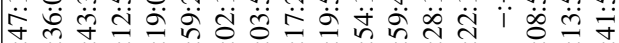

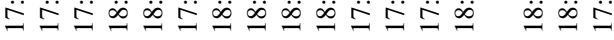

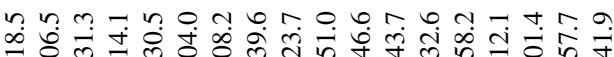

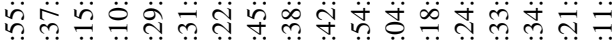

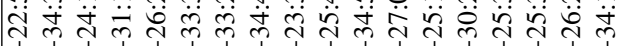

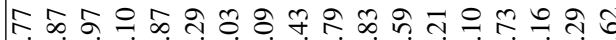

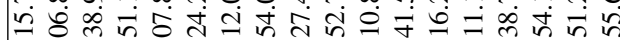

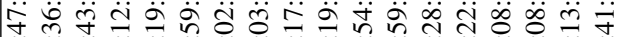

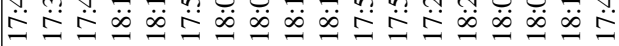

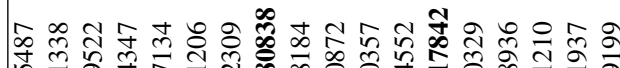

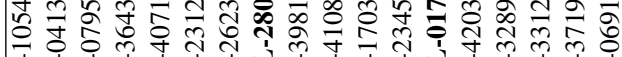

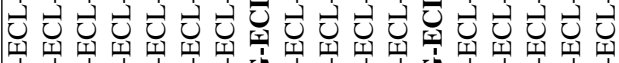

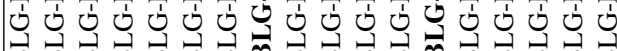
$m p m p m m m p m p m p m p$

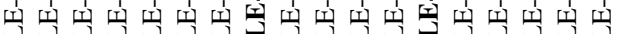

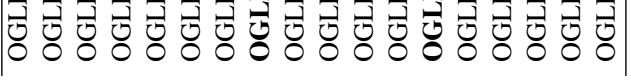




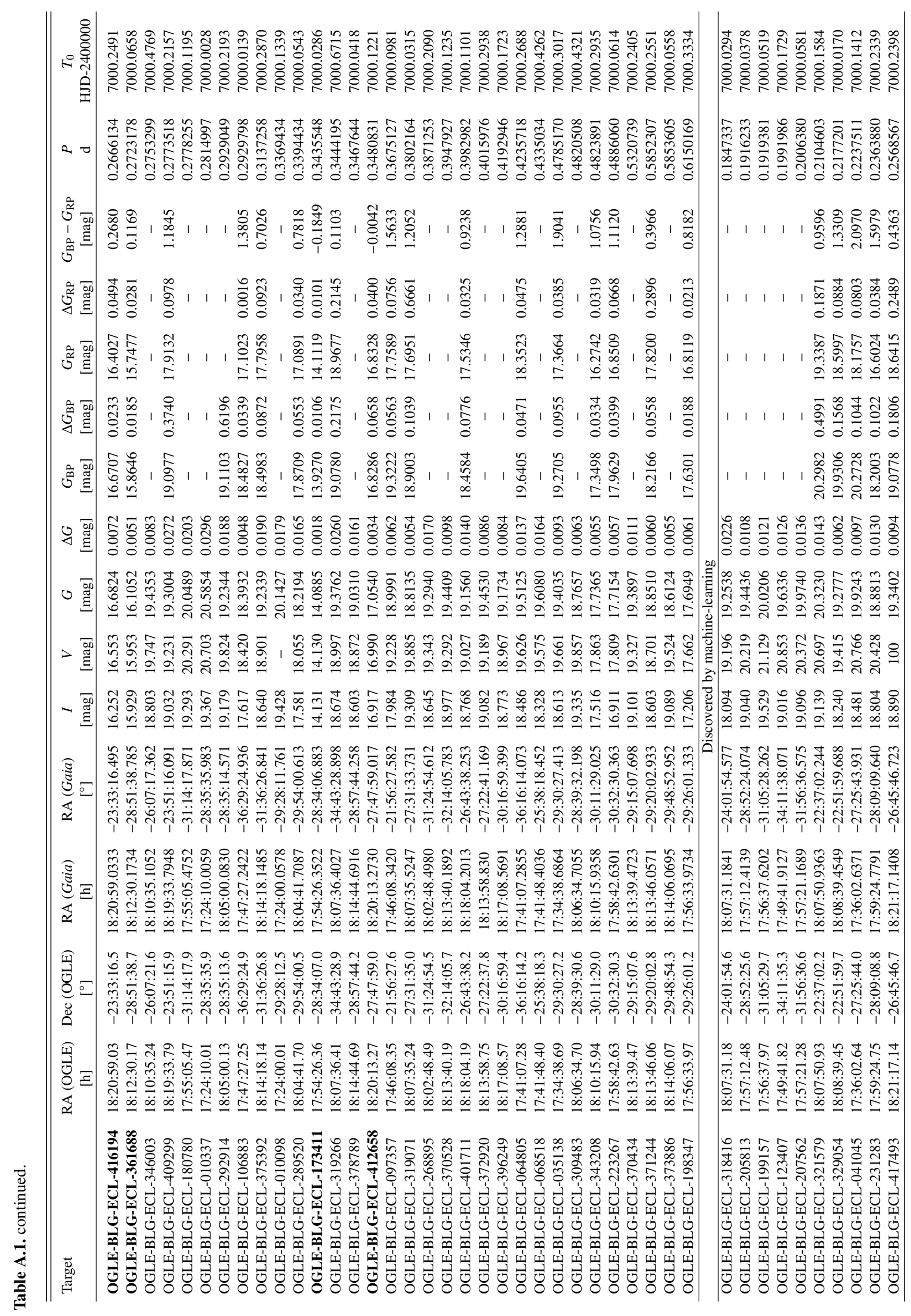




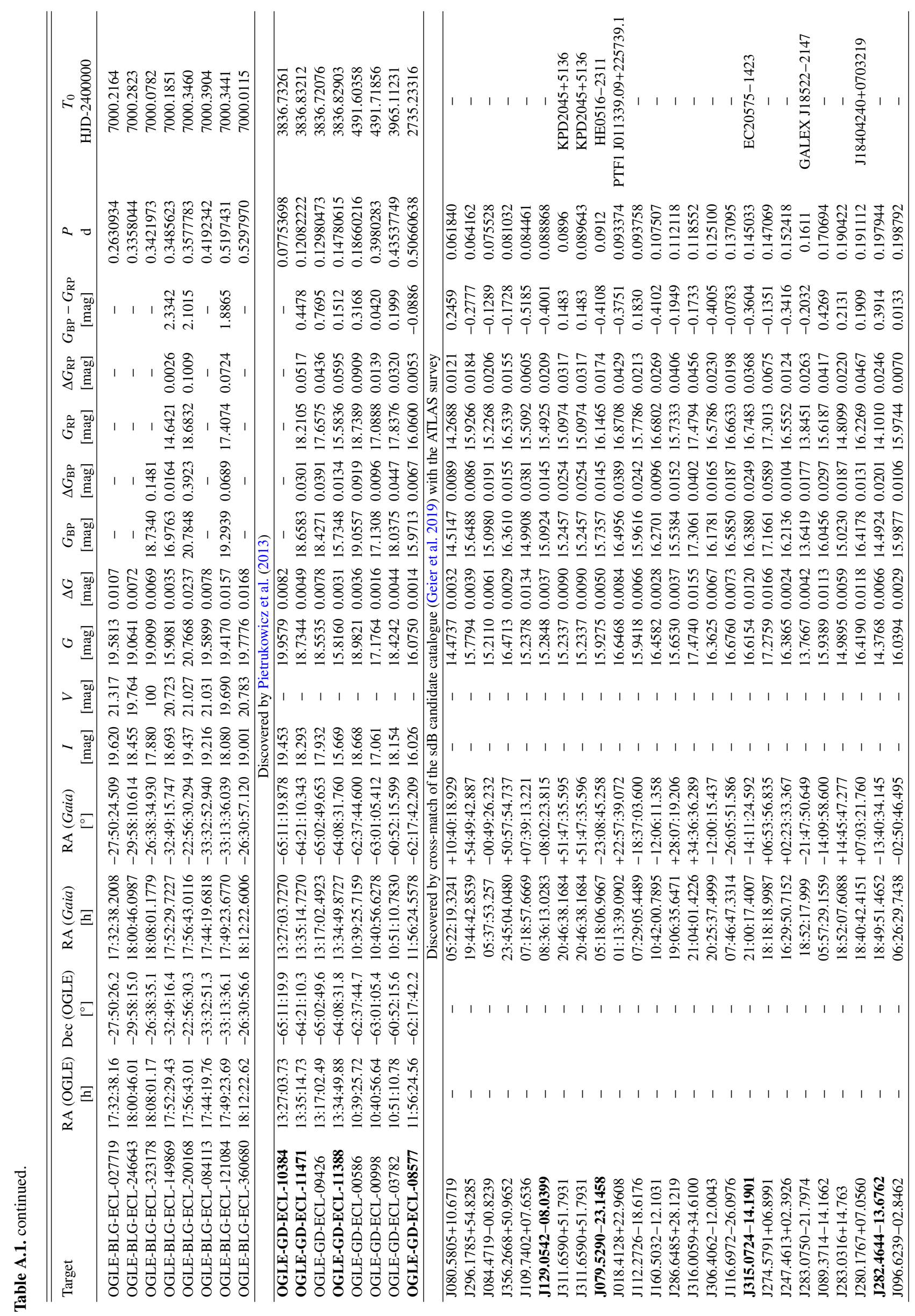




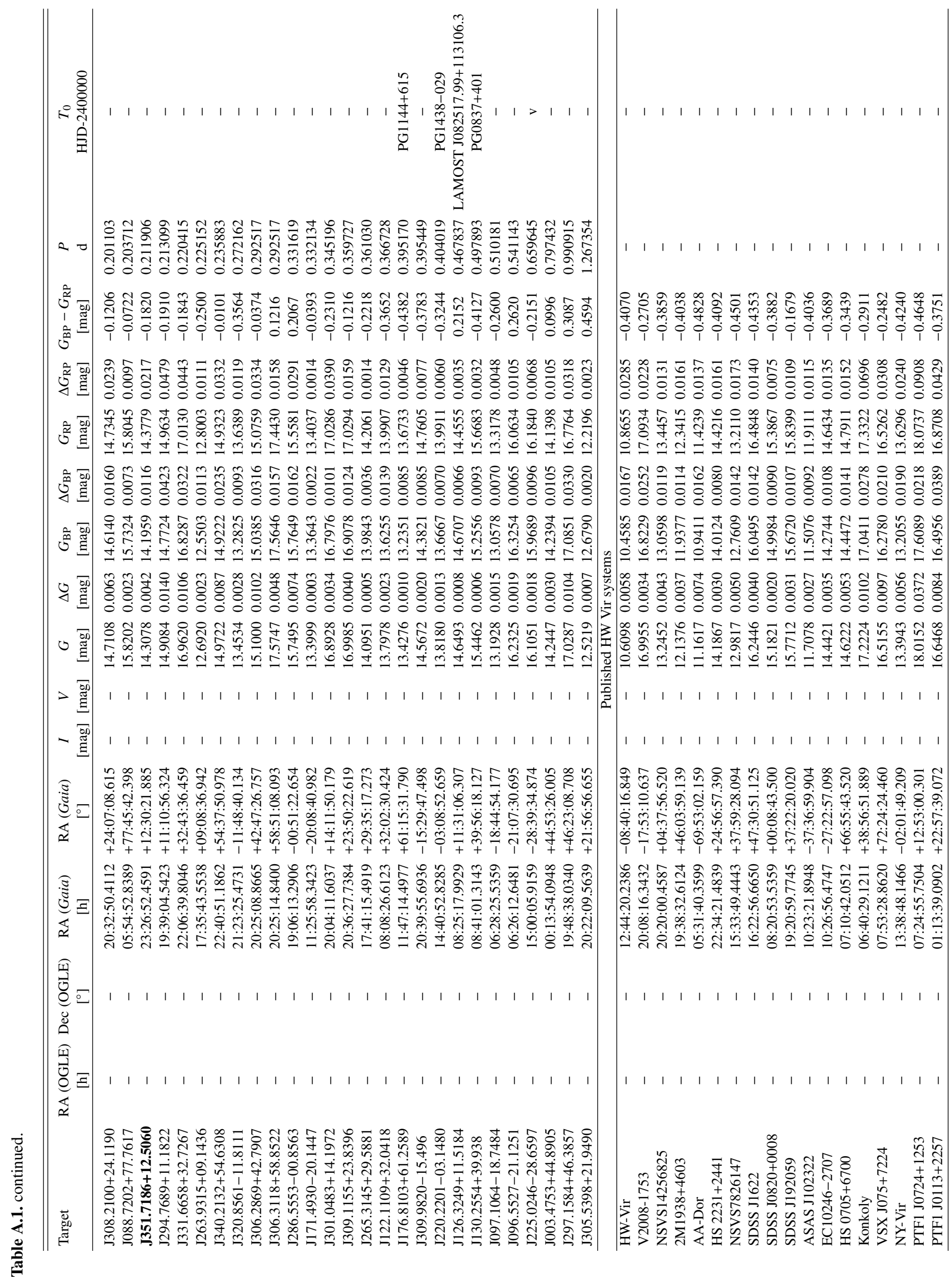


V. Schaffenroth et al.: The EREBOS project

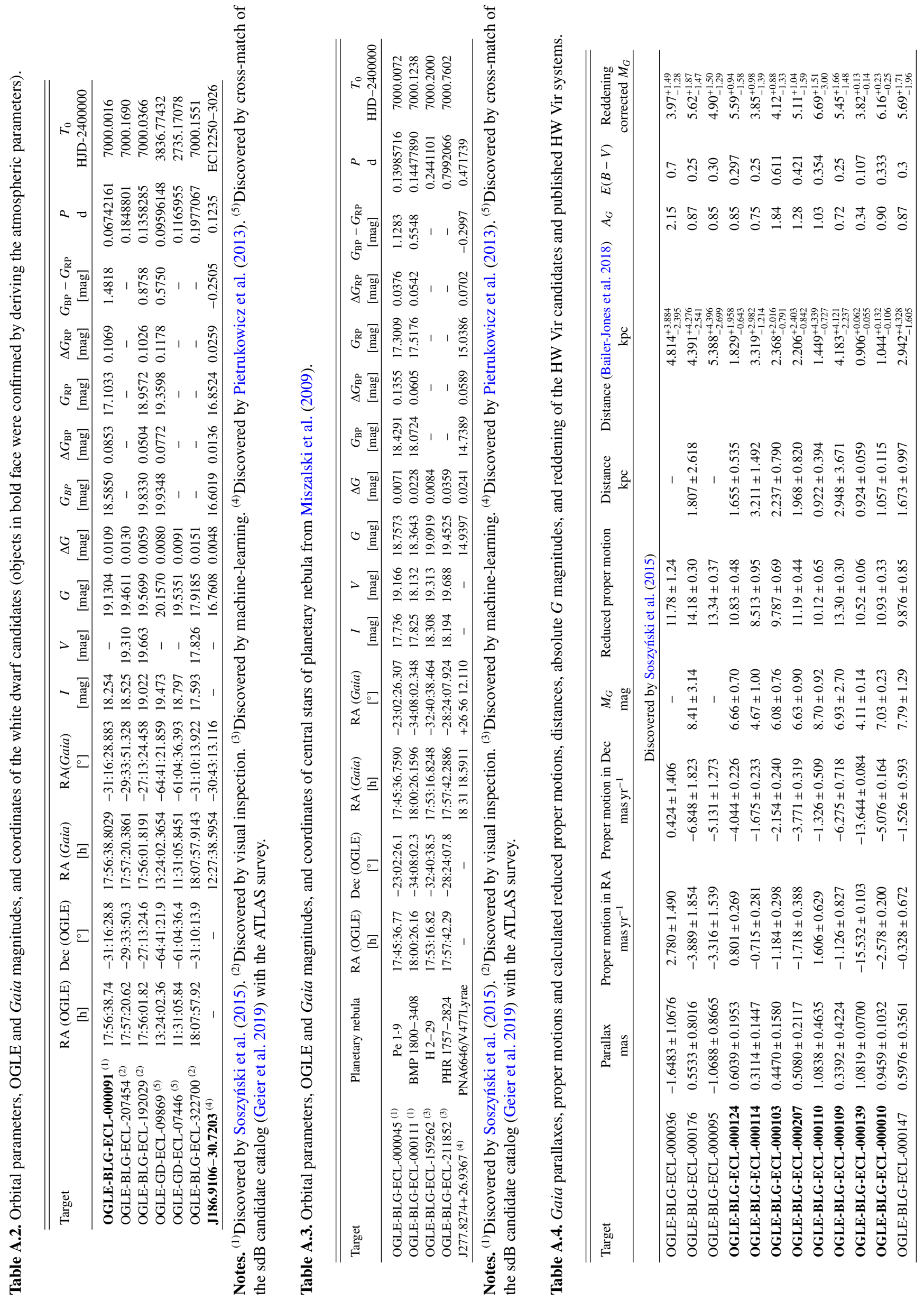




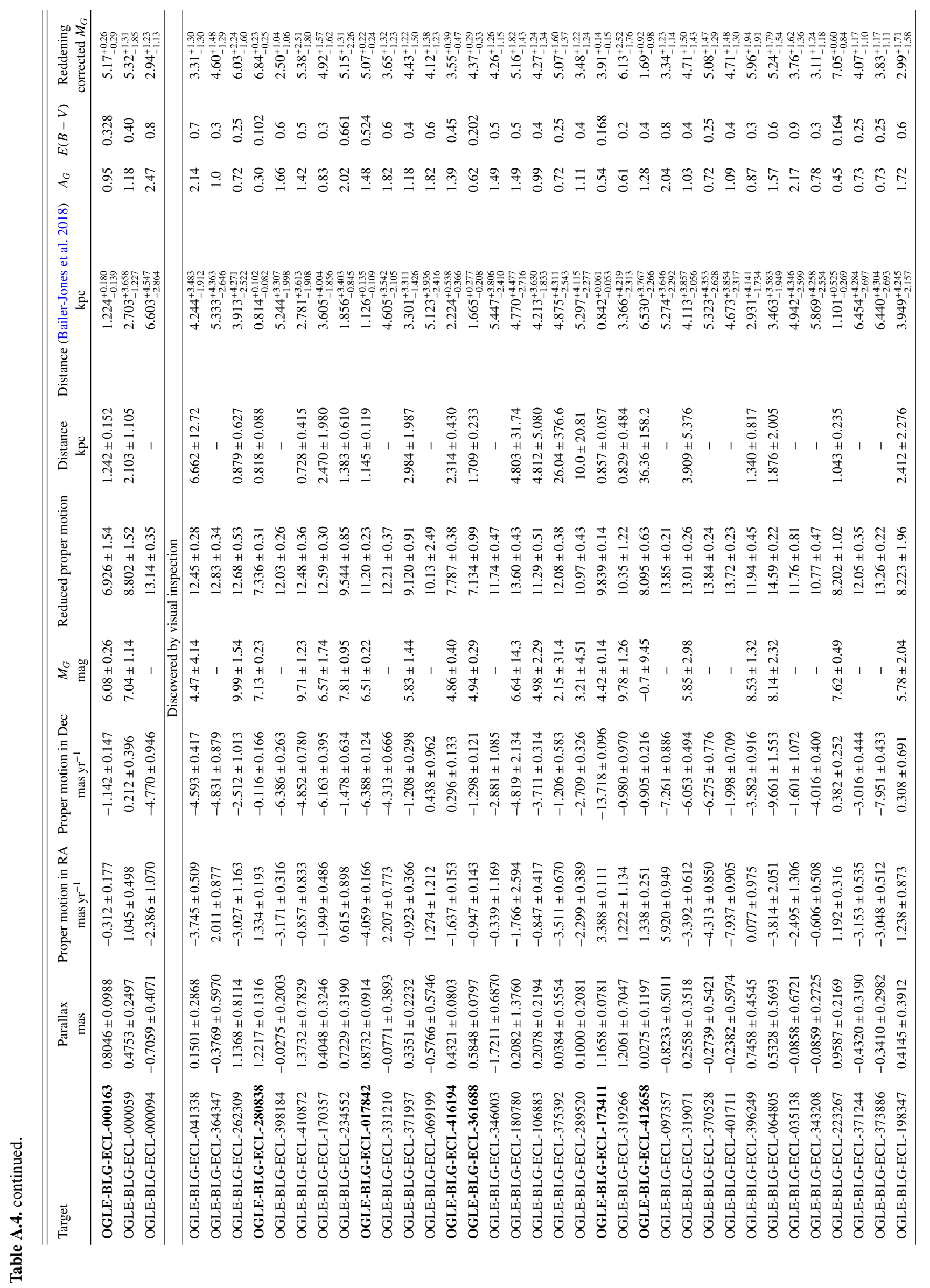




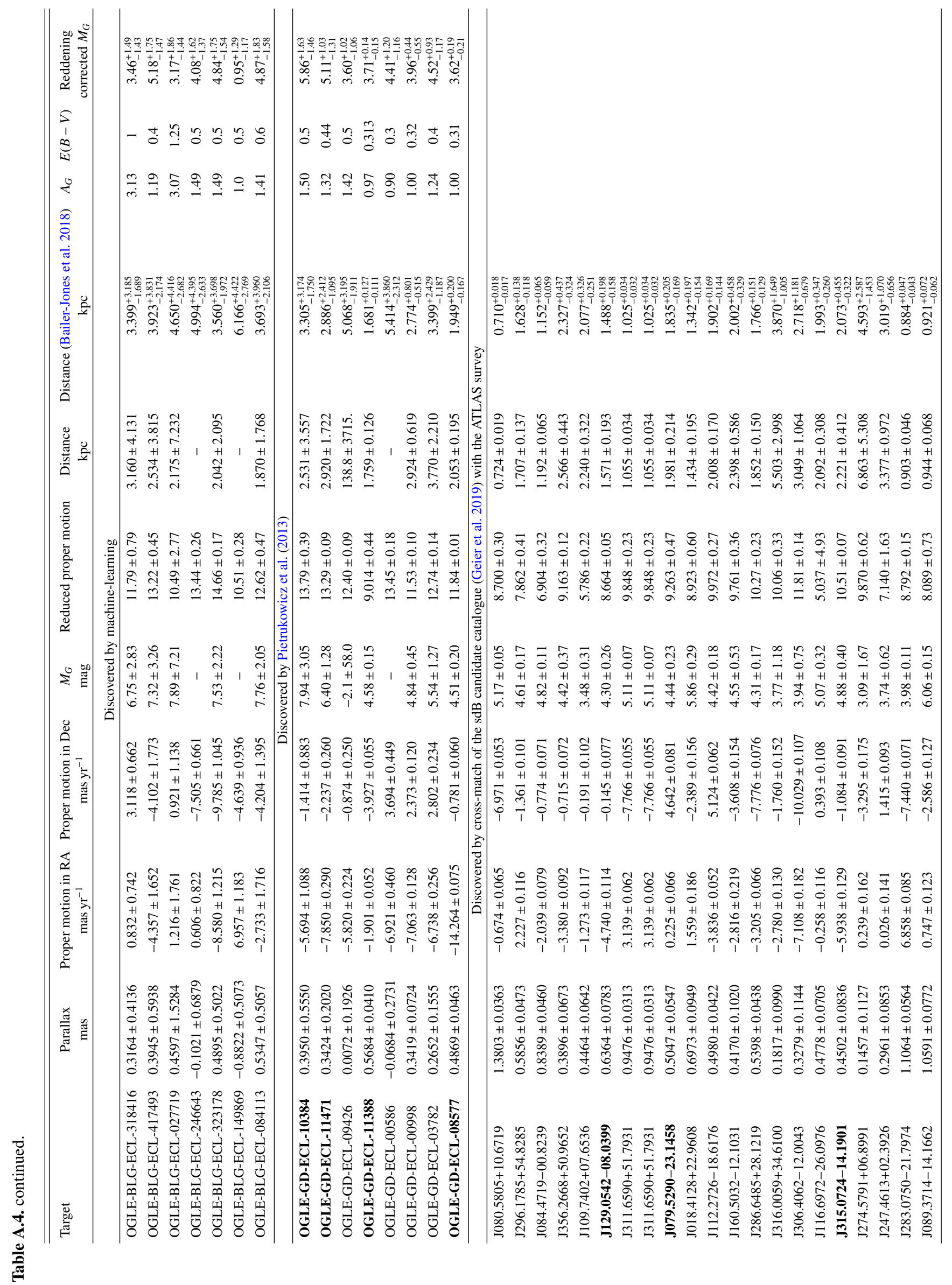




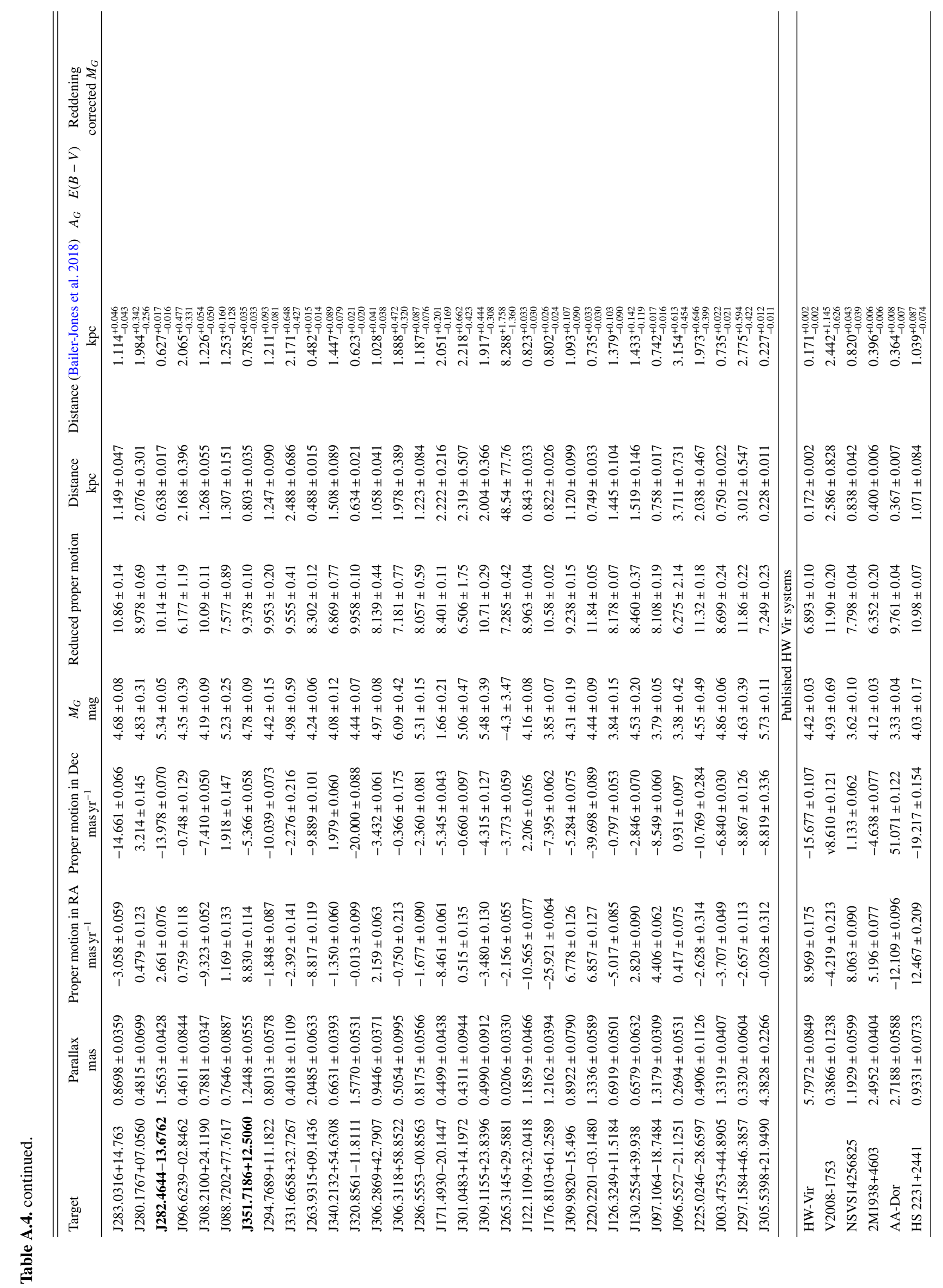



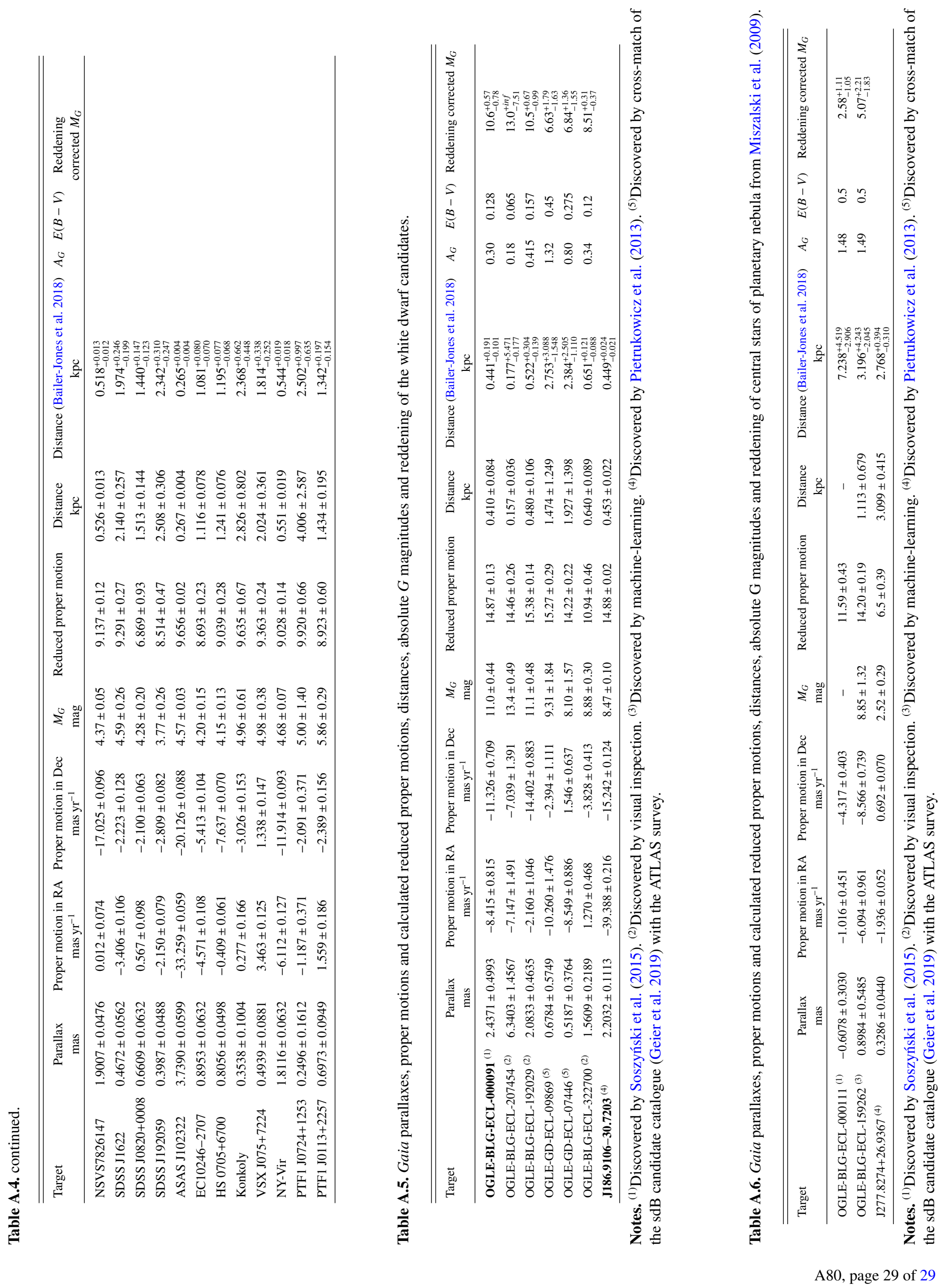